\title{
Convergence of conforming approximations for inviscid incompressible Bingham fluid flows and related problems
}

\author{
F. Bouchut, R. Eymard and A. Prignet*
}

September 9, 2013

\begin{abstract}
We study approximations by conforming methods of the solution to the variational inequality $\left\langle\partial_{t} u, v-u\right\rangle+\psi(v)-\psi(u) \geq\langle f, v-u\rangle$, which arises in the context of inviscid incompressible Bingham fluid flows and of the total variation flow problem. We propose a general framework involving total variation functionals, that enables to prove convergence of space, or time-space approximations, for steady or transient problems. We consider time implicit, or time implicit regularized (linearized or not) algorithms, and prove their convergence for general total variation functionals. Comparison with analytical solutions show the accuracy of the methods.
\end{abstract}

KEYwORDS. Total variation flow, Bingham fluids, conforming approximations, regularization method, convergence

\section{Introduction}

A series of practical physical and engineering problems involve the flows of the so-called incompressible "Bingham fluids". For such flows within a domain $\Omega \subset \mathbb{R}^{N}, N=2$ or 3 , the relation between the stress tensor $\sigma(t, x)$ (seen as a $N \times N$ matrix), the pressure $p(t, x)$ and the velocity $u(t, x) \in \mathbb{R}^{N}$ is given by (see for example [12] and references therein)

$$
\sigma=-p \mathrm{I}_{N}+\left(\frac{\kappa}{|D u|}+2 \nu\right) D u
$$

where $\kappa>0$ and $\nu>0$ are given physical coefficients ( $\nu$ is called the viscosity of the fluid), $\mathrm{I}_{N}$ is the $N \times N$ identity matrix, and where

$$
(D u)_{i j}=\frac{1}{2}\left(\partial_{i} u_{j}+\partial_{j} u_{i}\right), i, j=1, \ldots, N,
$$

denoting by $\partial_{i}$ the partial derivative with respect to the $i$-th coordinate of a point $x \in \Omega$, and

$$
|D u|^{2}=\sum_{i, j=1}^{N}(D u)_{i j}^{2} .
$$

\footnotetext{
${ }^{*}$ Université Paris-Est, Laboratoire d'Analyse et de Mathématiques Appliquées (UMR 8050), CNRS, UPEMLV, UPEC, F-77454, Marne-la-Vallée, France (francois.bouchut, robert.eymard, alain.prignet@univ-mlv.fr)
} 
Since the case of Bingham fluids with negligible viscosity arises in practice [23], our motivation is to provide here numerical methods which remain available in the case when $\nu$ is small. Therefore, this paper is focused on approximate methods allowing for the limit case $\nu=0$. The incompressibility condition for the fluid reads

$$
\operatorname{div} u=\sum_{i=1}^{N} \partial_{i} u_{i}=0 .
$$

Assuming a constant density $\rho=1$ for the fluid and neglecting the nonlinear convection term, the momentum conservation equation is given by

$$
\partial_{t} u_{i}-\sum_{j=1}^{N} \partial_{j} \sigma_{i j}=f_{i}, i=1, \ldots, N,
$$

where $f$ has values in $\mathbb{R}^{N}$. An initial condition

$$
u(0, \cdot)=u^{0}
$$

is considered, as well as boundary conditions. For simplicity, we assume here homogeneous Neumann boundary conditions, which can be written

$$
\sum_{j=1}^{N} \sigma_{i j} \mathbf{n}_{j}=0 \text { on } \partial \Omega, i=1, \ldots, N,
$$

where $\mathbf{n}$ is the outward normal unit vector on the boundary $\partial \Omega$ of $\Omega$.

In order to provide a formal variational formulation, let us define a set $E$ of regular functions $v: \Omega \rightarrow \mathbb{R}^{N}$ such that $\operatorname{div} v=0$. We then multiply (1.5) by $v_{i}$, sum over $i=1, \ldots, N$ and integrate on $\Omega$. We get, after an integration by parts in space accounting for the homogeneous Neumann boundary conditions and for the relation $\operatorname{div} v=0$,

$$
\forall v \in E, \int_{\Omega}\left(\partial_{t} u \cdot v+\left(\frac{\kappa}{|D u|}+2 \nu\right) D u: D v\right) \mathrm{d} x=\int_{\Omega} f \cdot v \mathrm{~d} x,
$$

where we denote by

$$
D u: D v=\sum_{i, j=1}^{N}(D u)_{i j}(D v)_{i j} .
$$

In (1.8) and (1.5), the ratio $D u /|D u|$ is not always defined. The physical understanding of this term indicates that it should be interpreted when $D u=0$ as "any trace-free symmetric matrix with norm less or equal to one". In the mathematical language such quantity is called "multivalued". This fundamental difficulty makes the approximation of the problem (1.8) already a complex challenge for $\nu>0$, and has motivated a large literature, see $[12,15,16]$ and references therein.

Since our motivation is to provide discretization methods also available in the case $\nu=0$, a first step is to rewrite (1.8) under a form which provides a well-posed continuous formulation. We then follow [12], writing a variational inequality giving a rigorous sense to this problem. Let us define, for $u, v \in E$ and $x \in \Omega$,

$$
A(u, v)(x)=D u(x): D v(x) \text { and } a(u)(x)=(A(u, u)(x))^{1 / 2} .
$$


Then from the Cauchy-Schwarz inequality one has

$$
A(u, v)+A(u, u)=A(u, u+v) \leq a(u) a(u+v),
$$

which gives

$$
\frac{1}{a(u)} A(u, v) \leq a(u+v)-a(u) .
$$

Let us also note that

$$
2 A(u, v) \leq A(u+v, u+v)-A(u, u) .
$$

Then, denoting

$$
\psi(v)=\int_{\Omega}(\kappa a(v)+\nu A(v, v)) \mathrm{d} x,
$$

the formulation (1.8) implies

$$
\forall v \in E, \quad \int_{\Omega} \partial_{t} u \cdot v \mathrm{~d} x+\psi(u+v)-\psi(u) \geq \int_{\Omega} f \cdot v \mathrm{~d} x .
$$

Reciprocally, letting $v=\theta w$ in the previous inequality, we note that

$$
A(u+\theta w, u+\theta w)-A(u, u)=\theta(2 A(u, w)+\theta A(w, w)),
$$

and

$$
a(u+\theta w)-a(u)=\theta \frac{2 A(u, w)+\theta A(w, w)}{a(u+\theta w)+a(u)} .
$$

We then formally recover (1.8) from (1.12) by dividing by $\theta$, letting $\theta>0$ tend to 0 and letting $\theta<0$ tend to 0 . We conclude that (1.12) is more general than (1.8) since it can be written in cases when $a(u)(x)=0$ occurs for some $x \in \Omega$. The formulation (1.12) can indeed be understood as saying that the linear form $v \mapsto \int\left(f-\partial_{t} u\right) \cdot v d x$ belongs to the subdifferential of the convex functional $\psi$ at $u$. The term in (1.8) appears in fact as the formal differential of the functional $\psi$.

Note that all the theoretical background of [12] relies on the viscous term, and strongly depends on the assumption $\nu>0$, while here we want to also handle the case $\nu=0$. We would like to extend the definition of $\psi(v)$ to all functions $v \in L^{2}(\Omega)^{N}$, thus we write for $v \in E$

$$
\begin{gathered}
\int_{\Omega}|D v| \mathrm{d} x=\sup _{\varphi \in\left(C_{c}^{1}(\Omega)\right)^{N^{2}},\|\varphi\|_{L^{\infty}(\Omega)} \leq 1} \int_{\Omega} D v: \varphi \mathrm{d} x, \\
\left(\int_{\Omega}|D v|^{2} \mathrm{~d} x\right)^{1 / 2}=\sup _{\varphi \in\left(C_{c}^{1}(\Omega)\right)^{N^{2}},\|\varphi\|_{L^{2}(\Omega)} \leq 1} \int_{\Omega} D v: \varphi \mathrm{d} x,
\end{gathered}
$$

where $C_{c}^{1}(\Omega)$ is the set of $C^{1}(\Omega)$ functions with compact support in $\Omega$. Integrating by parts, we thus extend the definition of $\psi(v)$ to all functions $v \in L^{2}(\Omega)^{N}$ by defining

$$
\psi(v)=\kappa \sup _{w \in V_{N, N}}\langle v, w\rangle+\nu\left(\sup _{w \in W_{N, N}}\langle v, w\rangle\right)^{2} \in[0, \infty],
$$


where $\langle\cdot, \cdot\rangle$ denotes the $L^{2}(\Omega)^{N}$ scalar product, and where

$$
\begin{gathered}
V_{N, N}=\left\{w \in L^{2}(\Omega)^{N}, \exists \varphi \in\left(C_{c}^{1}(\Omega)\right)^{N^{2}}, w_{i}=\frac{1}{2} \sum_{j=1}^{N} \partial_{j}\left(\varphi_{i j}+\varphi_{j i}\right), \sum_{i, j=1}^{N} \varphi_{i j}^{2} \leq 1 \text { in } \Omega\right\}, \\
W_{N, N}=\left\{w \in L^{2}(\Omega)^{N}, \exists \varphi \in\left(C_{c}^{1}(\Omega)\right)^{N^{2}}, w_{i}=\frac{1}{2} \sum_{j=1}^{N} \partial_{j}\left(\varphi_{i j}+\varphi_{j i}\right), \int_{\Omega} \sum_{i, j=1}^{N} \varphi_{i j}^{2} \mathrm{~d} x \leq 1\right\} .
\end{gathered}
$$

We consider the space $H$ of all functions $v \in L^{2}(\Omega)^{N}$ such that $\operatorname{div} v=0$. Then the functional $\psi$ may be defined by (1.15) as a mapping from $H$ to $[0, \infty]$, and we may define the set $B$ of all functions $v \in H$ such that $\psi(v)<\infty$. Indeed, the finiteness of the first term in (1.15) means that $D v$ is a finite measure on $\Omega$, while the finiteness of the second term (if $\nu>0$ ) means that $D v \in L^{2}(\Omega)$.

The problem is then to find, for a given $T>0$, a function $u$ such that

$$
\begin{gathered}
u \in L^{2}(0, T ; H), \int_{0}^{T} \psi(u(t)) \mathrm{d} t<\infty, \partial_{t} u \in L^{2}(0, T ; H), u(0)=u^{0} \text { and } \\
\forall v \in L^{2}(0, T ; H), \int_{0}^{T}\left(\left\langle\partial_{t} u(t), v(t)-u(t)\right\rangle+\psi(v(t))-\psi(u(t))\right) \mathrm{d} t \\
\geq \int_{0}^{T}\langle f(t), v(t)-u(t)\rangle \mathrm{d} t .
\end{gathered}
$$

For such nonlinear monotone variational inequalities in a Hilbert space, involving a convex lower semi-continuous functional (which is precisely the case for the function $\psi$ defined above and valued in $]-\infty, \infty]$ ), the theory of [6] applies, giving the existence and uniqueness of the solution.

This theory has been used in several works, applied to the total variation flow, and we refer to [22] for a general exposition on the subject. The total variation flow problem is a scalar problem which consists in looking for the solution $u: \Omega \rightarrow \mathbb{R}$ to the problem

$$
\partial_{t} u-\operatorname{div}\left(\frac{\nabla u}{|\nabla u|}\right)=f
$$

with Neumann boundary condition $(\nabla u /|\nabla u|) \cdot \mathbf{n}=0$ on $\partial \Omega$. Indeed, this problem may also be written under the form of inequality (1.18), denoting by $H$ the space $L^{2}(\Omega)$, and introducing the BV seminorm defined by

$$
\psi(v)=\sup _{w \in V_{N}}\langle v, w\rangle,
$$

where $\langle\cdot, \cdot\rangle$ denotes the $L^{2}(\Omega)$ scalar product and where

$$
V_{N}=\left\{w \in L^{2}(\Omega), \exists \varphi \in\left(C_{c}^{1}(\Omega)\right)^{N}, w=\operatorname{div} \varphi, \sum_{i=1}^{N} \varphi_{i}^{2} \leq 1 \text { in } \Omega\right\} .
$$

The total variation problem is however more "regular" than the inviscid Bingham problem, since it is also monotone in $L^{1}(\Omega)$, as proved in [1]. The theory of monotone problems in Banach spaces is provided in $[9,2,3]$. This $L^{1}$ monotonicity (implying the almost everywhere monotonicity, 
via the well-known Crandall-Tartar lemma [10]) enables to use the Kruzkov entropies, as for hyperbolic scalar conservation laws, and therefore to include a transport term. This is done in [4], including convergence results for numerical approximations.

The Bingham problem does not have this $L^{1}$ structure. Nevertheless, there is a very strong result in [20], that states that in two-dimensions, the Bingham evolution problem has a smooth solution, without viscosity, and including advection.

It is worth noticing that the form of inequality (1.18) includes both linear problems and nonlinear problems such as the $p$-Laplacian, which shows that it is quite general. Therefore it presents some interest to give general lines for its approximation.

This paper is devoted to the approximation by conforming methods of the solution to (1.18) in the general case of a convex lower semi-continuous (l.s.c. for short) functional on a Hilbert space. It is then applied to the framework of general total variation functionals, which includes the particular cases of the inviscid Bingham problem and of the total variation flow problem. In this situation we use a regularization procedure and time implicit or linearized implicit integration. Our results generalize the ones of $[13,14]$, obtained for the total variation flow, and for more regular data. Our analysis of the linearized implicit scheme, which is the one applied in practice for Bingham flows (see $[19,21]$ ), seems to be new.

The paper is organized as follows. In Section 2, we first recall some properties of the steady problem, following [6], and we propose sufficient conditions for a convergent approximation in a finite dimensional subspace, provided that some interpolation conditions be satisfied (Subsection 2.1). We then provide in Subsection 2.2 the analysis of the regularization method, applied to general total variation functionals. We turn to the transient problem in Section 3: we first state some basic properties in Subsection 3.1, and we then prove the convergence of a time-space conforming approximation of fully implicit type in Subsection 3.2. We provide in Subsection 3.3 the proof of convergence for the implicit regularization method for general total variation functionals, and treat the linearized algorithm in Subsection 3.4. We finally propose in Section 4 the study of numerical convergence in the particular case of the total variation flow. In a first subsection, we show that the problem to be solved in a finite dimensional space is itself approximated in the steady case. In a second subsection we consider the transient case, for which we show the convergence of the implicit method, where the use of a linearization is studied in the case of the regularized problem. Our results extend the ones of [13, 14], since they apply without further hypotheses on the regularity of the continuous solution. A short conclusion is finally given in Section 5.

\section{Approximation of the steady problem}

\subsection{General framework}

As stated in the introduction of this paper, we focus on the approximation of a steady version of the problem (1.18), using the framework of [6].

Let $H$ be a Hilbert space, with scalar product $\langle\cdot, \cdot\rangle$ and norm $\|\cdot\|$. Let $\psi: H \rightarrow]-\infty, \infty]$ be a convex, lower semi-continuous function such that the set $B=\{v \in H, \psi(v)<\infty\}$ (the domain of $\psi$ ) is not empty. Classical results in this situation can be found for example in [7].

We first recall the following standard lemma in convex analysis. 
Lemma 2.1 A functional $\psi$ is convex, lower semi-continuous with non-empty domain $B$, and first order positively homogeneous (i.e. for all $\lambda \in \mathbb{R}, v \in B, \psi(\lambda v)=|\lambda| \psi(v)$ ) if and only if there exists a non empty set $V \subset H$ such that

$$
\forall w \in V,-w \in V,
$$

and

$$
\forall v \in H, \psi(v)=\sup _{w \in V}\langle w, v\rangle .
$$

In this case, $B$ is a subspace of $H$, and $\psi$ satisfies $\psi(u+v) \leq \psi(u)+\psi(v)$.

Proof. It is given in [8] or [18, theorem 3.1.1], but for completeness we give it shortly. Since the "if" part is obvious, consider $\psi$ convex, lower semi-continuous with non-empty domain, and first order positively homogeneous. Then applying the homogeneity property to some $v_{0} \in B$ and $\lambda=0$ yields that $\psi(0)=0$. We deduce that $\psi(\lambda v)=|\lambda| \psi(v)$ for all $\lambda \in \mathbb{R}$ and $v \in H$, with the convention that $0 \times \infty=0$. In particular, $\psi(-v)=\psi(v)$. Applying the Fenchel-Moreau theorem we have that $\psi$ is the supremum of all affine functions upper dominated by $\psi$. Consider such an affine function $v \mapsto \mu+\langle w, v\rangle$ with $\mu \in \mathbb{R}$ and $w \in H$. We have

$$
\psi(v) \geq \mu+\langle w, v\rangle \text { for all } v \in H .
$$

Applying this inequality to $\lambda v$, using the homogeneity and letting $\lambda \rightarrow \infty$ yields that

$$
\psi(v) \geq\langle w, v\rangle \text { for all } v \in H .
$$

Applying this to $-v$ gives then that $\psi(v) \geq|\langle w, v\rangle| \geq 0$. Since (2.3) applied to $v=0$ gives $\mu \leq 0$, we deduce that the linear function $v \mapsto\langle w, v\rangle$, which is upper dominated by $\psi$ by (2.4), is greater than the affine function $v \mapsto \mu+\langle w, v\rangle$. Therefore, $\psi$ is also the supremum of all linear functions upper dominated by $\psi$. In other words, (2.2) holds with

$$
V=\{w \in H, \quad \forall v \in H\langle w, v\rangle \leq \psi(v)\} .
$$

It is easy to check finally that $0 \in V$ and that (2.1) holds.

Let us notice that the examples provided in the introduction of this paper (without viscosity) fall into the class given by Lemma 2.1.

We recall that, according to the convexity of $\psi$, the property of lower semi-continuity also holds for the weak topology of $H$, which implies that, for any sequence $\left(v_{n}\right)_{n \in \mathbb{N}}$ of elements of $B$ that weakly converges to $v \in H$, and such that there exists $C \in \mathbb{R}$ with $\psi\left(v_{n}\right) \leq C$ for all $n \in \mathbb{N}$, then $\psi(v) \leq \liminf _{n} \psi\left(v_{n}\right)$, which implies that $v \in B$.

Let $\alpha>0$ and $f \in H$ be given. The aim of this section is to approximate the solution to the following problem: find $u$ such that

$$
\begin{aligned}
& u \in B, \\
& \forall v \in B, \quad \alpha\langle u, v-u\rangle+\psi(v)-\psi(u) \geq\langle f, v-u\rangle .
\end{aligned}
$$

Note that (2.6) may also be written as

$$
\alpha u+\partial \psi(u) \ni f
$$


with introducing the subdifferential of the function $\psi$ at $u$,

$$
\partial \psi(u)=\{w \in H, \forall v \in H, \psi(v) \geq \psi(u)+\langle w, v-u\rangle\} .
$$

Then according to [6] we have the following result.

Lemma 2.2 There is existence and uniqueness of the solution $u$ to the problem (2.6), which moreover satisfies

$$
u=\underset{v \in B}{\operatorname{argmin}} J(v)
$$

where $J: B \rightarrow \mathbb{R}$ is defined by

$$
J(v)=\frac{\alpha}{2}\|v\|^{2}+\psi(v)-\langle f, v\rangle, \forall v \in B
$$

Corollary 2.3 There exists $u_{0} \in B$ such that $\partial \psi\left(u_{0}\right)$ is not empty.

Proof. Take $\alpha=1$ and $f=0$. Then the solution $u$ obtained by Lemma 2.2 satisfies (2.7), which implies that $\partial \psi(u)$ is not empty.

Remark 2.4 In the case when $\psi$ is first order positively homogeneous (the case of Lemma 2.1), then $u \in B$ is solution to the problem (2.6) if and only if

$$
\begin{aligned}
& u \in H \\
& \forall v \in H, \quad \alpha\langle u, v\rangle+\psi(v) \geq\langle f, v\rangle,
\end{aligned}
$$

and

$$
\alpha\|u\|^{2}+\psi(u)=\langle f, u\rangle .
$$

It indeed suffices to let $v=0$ and $v=2 u$ in (2.6) for obtaining (2.12), which also shows that $u \in B$. This characterization is used in the examples for exhibiting an analytical solution.

Remark 2.5 If we have two solutions $u_{1}$ and $u_{2}$ to (2.6) associated to two different right-hand sides $f_{1}$ and $f_{2}$, then one has $\left\|u_{2}-u_{1}\right\| \leq\left\|f_{2}-f_{1}\right\| / \alpha$. This is obtained by taking $v=u_{2}$ in the formulation (2.6) for $u_{1}$, taking $v=u_{1}$ in the formulation (2.6) for $u_{2}$, and adding the results. This contraction property is in the heart of the theory of monotone operators (here $\partial \psi$ is the monotone operator in (2.7)).

Let us now introduce a reduction argument. Take $u_{0} \in B$ such that $\partial \psi\left(u_{0}\right)$ is not empty, and pick some $w \in \partial \psi\left(u_{0}\right)$. Then one has $\psi(v) \geq \psi\left(u_{0}\right)+\left\langle w, v-u_{0}\right\rangle$ for all $v \in H$. Therefore, setting for $v \in H$

$$
\widetilde{\psi}(v)=\psi\left(v+u_{0}\right)-\psi\left(u_{0}\right)-\langle w, v\rangle,
$$

the functional $\widetilde{\psi}: H \rightarrow]-\infty, \infty]$ is convex and lower semi-continuous, and satisfies $\widetilde{\psi} \geq 0$, and $\widetilde{\psi}(0)=0$ (implying $0 \in \widetilde{B}=\{v \in H, \widetilde{\psi}(v)<\infty\}$ ). For $a \in \mathbb{R}$ (chosen later) and for $v \in H$, define then

$$
\widetilde{J}(v)=J\left(v+u_{0}\right)-a=\frac{\alpha}{2}\left\|v+u_{0}\right\|^{2}+\psi\left(v+u_{0}\right)-\left\langle f, v+u_{0}\right\rangle-a .
$$


Then the minimum of $\widetilde{J}$ is obtained at the point $\widetilde{u}=u-u_{0}$, where $u$ is the solution to the problem (2.6). Setting $\widetilde{f}=f-w-\alpha u_{0}$, we have

$$
\widetilde{J}(v)=\frac{\alpha}{2}\|v\|^{2}+\widetilde{\psi}(v)-\langle\widetilde{f}, v\rangle+\psi\left(u_{0}\right)+\left\langle\frac{\alpha}{2} u_{0}-f, u_{0}\right\rangle-a,
$$

thus choosing $a=\psi\left(u_{0}\right)+\left\langle\frac{\alpha}{2} u_{0}-f, u_{0}\right\rangle$, the problem is to find the minimum of

$$
\widetilde{J}(v)=\frac{\alpha}{2}\|v\|^{2}+\widetilde{\psi}(v)-\langle\widetilde{f}, v\rangle .
$$

Therefore, $\widetilde{u}$ is the solution to the problem

$$
\begin{aligned}
& \widetilde{u} \in \widetilde{B}, \\
& \forall v \in \widetilde{B}, \quad \alpha\langle\widetilde{u}, v-\widetilde{u}\rangle+\widetilde{\psi}(v)-\widetilde{\psi}(\widetilde{u}) \geq\langle\widetilde{f}, v-\widetilde{u}\rangle .
\end{aligned}
$$

Indeed, (2.14) can also be deduced directly from (2.6). We conclude that we may assume, without loss of generality, that $\psi \geq 0$ and $\psi(0)=0$. This is done in the following Hypothesis.

Hypothesis 2.6 We consider the following assumptions:

1. $H$ is a Hilbert space,

2. $\psi: H \rightarrow]-\infty, \infty]$ is convex and lower semi-continuous,

3. $\psi(v) \geq 0$ for all $v \in H$, and $\psi(0)=0$.

Note that the third assumption ensures that if $f=0$, the solution to (2.6) is $u=0$.

Assuming Hypothesis 2.6 (that implies $0 \in B=\{v \in H, \psi(v)<\infty\}$ ), let $\widehat{H}$ be a closed subspace of $H$ and let $\widehat{B}=\widehat{H} \cap B$. Note that $0 \in \widehat{B}$ (in the examples, $B$ is a subspace of $H$ and $\widehat{H}$ is a finite dimensional subspace of $H$ ). We define the approximate problem as: find $\widehat{u}$ such that

$$
\begin{aligned}
& \widehat{u} \in \widehat{B}, \\
& \forall v \in \widehat{B}, \quad \alpha\langle\widehat{u}, v-\widehat{u}\rangle+\psi(v)-\psi(\widehat{u}) \geq\langle f, v-\widehat{u}\rangle .
\end{aligned}
$$

It is then immediate to get the existence and uniqueness of $\widehat{u}$ solution to (2.15).

Lemma 2.7 Under Hypothesis 2.6, let $\alpha>0$ and $f \in H$ be given. Let $\widehat{H}$ be a closed subspace of $H$ and let $\widehat{B}=\widehat{H} \cap B$. Then there exists one and only one solution to the problem (2.15), which moreover satisfies

$$
\widehat{u}=\underset{v \in \widehat{B}}{\operatorname{argmin}} J(v),
$$

where $J$ is defined by (2.10).

Proof. It suffices to consider $\widehat{f}$, the orthogonal projection of $f$ on $\widehat{H}$. Then $(2.15)$ is identical to $(2.6)$ replacing $\psi$ by its restriction to $\widehat{H}$ and $f$ by $\widehat{f}$, since $\langle\widehat{f}, v\rangle=\langle f, v\rangle$ for all $v \in \widehat{H}$. Therefore, Lemma 2.2 gives the existence and uniqueness of the solution to (2.15).

The scheme (2.15) leads to the resolution of a convex minimization problem in a finite dimensional space. There are many numerical methods well-suited to that (gradient or conjugate gradient methods for example). We analyze a different type of method, the regularization method, in the particular case of total variation functionals in Subsection 2.2, based on the particular form of the function $\psi$. Let us however give the following error estimate result for the scheme (2.15) in the general case. 
Lemma 2.8 Under Hypothesis 2.6, let $\alpha>0$ and $f \in H$ be given and let $u \in B$ be the solution to the problem (2.6). Let $\widehat{H}$ be a closed subspace of $H$, let $\widehat{B}=\widehat{H} \cap B$ and let $\widehat{u} \in \widehat{B}$ be the solution to (2.15). Then

$$
\|u-\widehat{u}\| \leq 2\left(\frac{\widehat{R}_{u, f}}{\alpha}\right)^{1 / 2},
$$

and

$$
|\psi(u)-\psi(\widehat{u})| \leq 6\|f\|\left(\frac{\widehat{R}_{u, f}}{\alpha}\right)^{1 / 2},
$$

with

$$
\widehat{R}_{u, f}=\inf _{v \in \widehat{B}}\left(\|f\|\|v-u\|+(\psi(v)-\psi(u))^{+}\right),
$$

where we denote for all $x \in \mathbb{R}, x^{+}=\max (0, x)$.

Proof. Let us first observe that $\widehat{u}$ satisfies, setting $v=0$ in (2.15) and using that $\psi(0)=0$,

$$
\alpha\|\widehat{u}\|^{2}+\psi(\widehat{u}) \leq\langle f, \widehat{u}\rangle,
$$

which gives, according to the Young inequality,

$$
\frac{\alpha}{2}\|\widehat{u}\|^{2}+\psi(\widehat{u}) \leq \frac{1}{2 \alpha}\|f\|^{2} .
$$

Since $\psi \geq 0$ this shows that

$$
\|\widehat{u}\| \leq \frac{1}{\alpha}\|f\| .
$$

We similarly write, from taking $v=0$ in $(2.6)$,

$$
\|u\| \leq \frac{1}{\alpha}\|f\| .
$$

From (2.15), we get for any $v \in \widehat{B}$

$$
\alpha\langle\widehat{u}, u-\widehat{u}\rangle+\psi(u)-\psi(\widehat{u})+R(v) \geq\langle f, u-\widehat{u}\rangle,
$$

with

$$
R(v)=\alpha\langle\widehat{u}, v-u\rangle+\psi(v)-\psi(u)+\langle f, u-v\rangle .
$$

Hence, taking the infimum, we get

$$
\alpha\langle\widehat{u}, u-\widehat{u}\rangle+\psi(u)-\psi(\widehat{u})+\inf _{v \in \widehat{B}} R(v) \geq\langle f, u-\widehat{u}\rangle,
$$

while from (2.23) and (2.19) we have

$$
\inf _{v \in \widehat{B}} R(v) \leq 2 \widehat{R}_{u, f}
$$

We have also, letting $v=0$ in (2.23) and using that $\psi(0)=0, \psi \geq 0$ and (2.21)-(2.22),

$$
\inf _{v \in \widehat{B}} R(v) \leq R(0) \leq \frac{2}{\alpha}\|f\|^{2} .
$$


Taking $v=\widehat{u}$ in $(2.6)$, we get

$$
\alpha\langle u, \widehat{u}-u\rangle+\psi(\widehat{u})-\psi(u) \geq\langle f, \widehat{u}-u\rangle .
$$

Adding the inequalities (2.24) and (2.27) yields

$$
\alpha\|u-\widehat{u}\|^{2} \leq \inf _{v \in \widehat{B}} R(v),
$$

which provides (2.17), using (2.25). We deduce also that the right-hand side of (2.28) is nonnegative. We then write, again using (2.24) and (2.21),

$$
\psi(\widehat{u})-\psi(u) \leq 2\|f\|\|u-\widehat{u}\|+\inf _{v \in \widehat{B}} R(v),
$$

and similarly from $(2.27)$

$$
\psi(u)-\psi(\widehat{u}) \leq \alpha\langle u, \widehat{u}-u\rangle+\langle f, u-\widehat{u}\rangle \leq 2\|f\|\|u-\widehat{u}\| .
$$

We deduce, with (2.28) and (2.26), that

$$
\begin{aligned}
|\psi(u)-\psi(\widehat{u})| & \leq 2\|f\|\|u-\widehat{u}\|+\inf _{v \in \widehat{B}} R(v) \\
& \leq \sqrt{\inf _{v \in \widehat{B}} R(v)}\left(\frac{2\|f\|}{\sqrt{\alpha}}+\sqrt{\inf _{v \in \widehat{B}} R(v)}\right) \\
& \leq \frac{4\|f\|}{\sqrt{\alpha}} \sqrt{\inf _{v \in \widehat{B}} R(v)},
\end{aligned}
$$

which leads to (2.18) using (2.25).

We deduce the following convergence result for the approximation method.

Corollary 2.9 Under Hypothesis 2.6, let $\left(\widehat{H}_{n}\right)_{n \in \mathbb{N}}$ be a sequence of closed subspaces of $H$, and let, for all $n \in \mathbb{N}, \widehat{B}_{n}=\widehat{H}_{n} \cap B$. We assume that

$$
\lim _{n \rightarrow \infty} \inf _{w \in \widehat{B}_{n}}\left(\|w-v\|+(\psi(w)-\psi(v))^{+}\right)=0, \forall v \in B .
$$

Let, for all $n \in \mathbb{N}, \widehat{u}_{n} \in \widehat{B}_{n}$ be the unique solution $\widehat{u}$ to (2.15) with $\widehat{B}=\widehat{B}_{n}$. Then, $\widehat{u}_{n}$ converges in $H$ to the unique solution $u \in B$ to the problem (2.6) as $n$ tends to $\infty$ and $\psi\left(\widehat{u}_{n}\right)$ converges to $\psi(u)$.

\subsection{Total variation functionals}

In this subsection we apply the framework of the previous section to the case of functionals $\psi$ of total variation type, generalizing (1.20), or (1.15) with $\nu=0$, in the introduction of this paper.

Hypothesis 2.10 We assume Hypothesis 2.6. Moreover, we assume that there exists a subspace $H_{1} \subset H$, an open set $\Omega \subset \mathbb{R}^{N}$ with $N \geq 1$, a nonnegative Borel measure $\mu$ on $\Omega$ such that 
$\mu(\Omega)<\infty$, and a symmetric bilinear mapping $A: H_{1} \times H_{1} \rightarrow L_{\mu}^{1}(\Omega)$ such that $A(u, u)(x) \geq 0$ for $\mu$ a.e. $x \in \Omega$, for all $u \in H_{1}$, and

$$
\forall u \in H_{1}, \psi(u)=\int_{\Omega} a(u)(x) \mathrm{d} \mu,
$$

denoting by $a(u)(x)=(A(u, u)(x))^{1 / 2}$ for $\mu$ a.e. $x \in \Omega$. In particular, $\psi$ is finite on $H_{1}$, i.e. $H_{1} \subset B$.

The two examples we are interested in obviously satisfy these conditions, with the following choices.

Example 2.11 (Total variation flow) The Hilbert space is taken $H=L^{2}(\Omega)$, with $\Omega$ a bounded open set in $\mathbb{R}^{N}, \psi$ is as in (1.20), (1.21), $B=L^{2}(\Omega) \cap B V(\Omega)$, $\mu$ is the Lebesgue measure on $\Omega, H_{1}$ is any space such that $C^{\infty}(\bar{\Omega}) \subset H_{1} \subset H^{1}(\Omega)$, and $A(u, v)(x)=\nabla u(x) \cdot \nabla v(x)$.

Example 2.12 (Inviscid Bingham flow) The Hilbert space is taken $H=\left\{u \in L^{2}(\Omega)^{N}\right.$, $\operatorname{div} u=$ $0\}$ with $\Omega$ a bounded open set in $\mathbb{R}^{N}, \psi$ is as in (1.15), (1.16) with $\nu=0, B=\{u \in$ $\left.L^{2}(\Omega)^{N}, \operatorname{div} u=0, D u \in \mathcal{M}(\Omega)\right\}$, where $D u=\left(\nabla u+(\nabla u)^{t}\right) / 2, \mathcal{M}(\Omega)$ is the space of $f$ nite measures over $\Omega, \mu$ is $\kappa$ times the Lebesgue measure on $\Omega, H_{1}$ is any space such that $\left\{u \in C^{\infty}(\bar{\Omega})^{N}, \operatorname{div} u=0\right\} \subset H_{1} \subset\left\{u \in H^{1}(\Omega)^{N}, \operatorname{div} u=0\right\}$, and $A(u, v)(x)=D u(x): D v(x)$.

We next analyze the algorithm by regularization for computing an approximate solution to (2.16) (which converges to the continuous solution according to Corollary 2.9).

Lemma 2.13 Under Hypothesis 2.10, let $\alpha>0$ and $f \in H$. Let $\widehat{H}$ be a finite dimensional subspace of $H_{1}$. Then, for $\varepsilon>0$, there exists one and only one function $\widehat{u}_{\varepsilon} \in \widehat{H}$ solution to

$$
\begin{aligned}
& \widehat{u}_{\varepsilon} \in \widehat{H}, \\
& \forall v \in \widehat{H}, \alpha\left\langle\widehat{u}_{\varepsilon}, v\right\rangle+\int_{\Omega} \frac{A\left(\widehat{u}_{\varepsilon}, v\right)(x)}{\varepsilon+a\left(\widehat{u}_{\varepsilon}\right)(x)} \mathrm{d} \mu=\langle f, v\rangle .
\end{aligned}
$$

Moreover, denoting by $\widehat{u} \in \widehat{H}$ the unique solution to (2.16), we have

$$
\left\|\widehat{u}_{\varepsilon}-\widehat{u}\right\| \leq \sqrt{\frac{\mu(\Omega) \varepsilon}{\alpha}},
$$

and

$$
\left|\psi\left(\widehat{u}_{\varepsilon}\right)-\psi(\widehat{u})\right| \leq 2\|f\| \sqrt{\frac{\mu(\Omega) \varepsilon}{\alpha}}+3 \mu(\Omega) \varepsilon .
$$

The estimates (2.32), (2.33) provide, with Corollary 2.9, the convergence of $\left(\widehat{u}_{\varepsilon}\right)_{n}$ to $u$ under the conditions (2.29) and $\varepsilon \rightarrow 0$. Note that since $\widehat{H}_{n} \subset H_{1}$, it is necessary for having (2.29) that the following condition holds:

$$
\forall v \in B, \inf _{w \in H_{1}}\left(\|w-v\|+(\psi(w)-\psi(v))^{+}\right)=0 .
$$

This condition is proved to hold true for the total variation flow and for the Bingham flow in the appendix. Then to recover (2.29) from (2.34), it suffices to require that

$$
\lim _{n \rightarrow \infty} \inf _{w \in \widehat{H}_{n}}\left(\|w-v\|+(\psi(w)-\psi(v))^{+}\right)=0, \forall v \in H_{1},
$$


which is easily fulfilled.

Proof of Lemma 2.13. Let us begin with the proof of uniqueness. Consider two elements $u_{1}$ and $u_{2}$ of $\widehat{H}$ satisfying (2.31). Subtracting (2.31) with $u_{1}$ and $u_{2}$, and taking $v=u_{1}-u_{2}$ leads to

$$
\alpha\left\|u_{1}-u_{2}\right\|^{2}+\int_{\Omega}\left(\frac{A\left(u_{1}, u_{1}-u_{2}\right)(x)}{\varepsilon+a\left(u_{1}\right)(x)}-\frac{A\left(u_{2}, u_{1}-u_{2}\right)(x)}{\varepsilon+a\left(u_{2}\right)(x)}\right) \mathrm{d} \mu=0 .
$$

We have according to the Cauchy-Schwarz inequality $A\left(u_{1}, u_{2}\right)(x) \leq a\left(u_{1}\right)(x) a\left(u_{2}\right)(x)$ for $\mu$ a.e. $x \in \Omega$. Since $s \rightarrow s /(\varepsilon+s)$ is strictly increasing from $[0, \infty)$ to $[0,1)$, omitting for simplicity the argument $x$ we get

$$
\begin{aligned}
0 & \leq\left(\frac{a\left(u_{1}\right)}{\varepsilon+a\left(u_{1}\right)}-\frac{a\left(u_{2}\right)}{\varepsilon+a\left(u_{2}\right)}\right)\left(a\left(u_{1}\right)-a\left(u_{2}\right)\right) \\
& \leq\left(\frac{A\left(u_{1}, u_{1}-u_{2}\right)}{\varepsilon+a\left(u_{1}\right)}-\frac{A\left(u_{2}, u_{1}-u_{2}\right)}{\varepsilon+a\left(u_{2}\right)}\right) .
\end{aligned}
$$

Using this information in (2.36) we obtain

$$
\alpha\left\|u_{1}-u_{2}\right\|^{2} \leq 0
$$

which concludes the proof of uniqueness. Turning to the existence proof, we consider the operator $T: \widehat{H} \rightarrow \widehat{H}$ defined for $u \in \widehat{H}$ by

$$
\forall v \in \widehat{H},\langle T(u), v\rangle=\int_{\Omega} \frac{A(u, v)(x)}{\varepsilon+a(u)(x)} \mathrm{d} \mu .
$$

Since $A$ is bilinear and $\widehat{H}$ is finite dimensional, the restriction of $A$ to $\widehat{H} \times \widehat{H}$ is continuous, with values in $L_{\mu}^{1}(\Omega)$. Therefore, the operator $T$ is continuous from $\widehat{H}$ to itself. Then, we have that any solution to $(2.31)$, in which a factor $\lambda \in[0,1]$ is introduced in front of the integral, satisfies the estimate

$$
\frac{\alpha}{2}\left\|\widehat{u}_{\varepsilon}\right\|^{2}+\lambda \int_{\Omega} \frac{a\left(\widehat{u}_{\varepsilon}\right)(x)^{2}}{\varepsilon+a\left(\widehat{u}_{\varepsilon}\right)(x)} \mathrm{d} \mu \leq \frac{1}{2 \alpha}\|f\|^{2} .
$$

Since, for $\lambda=0$, the problem is a finite dimensional invertible linear problem, we get by a standard topological degree argument that there exists at least one solution to the problem for $\lambda=1$. Let us now turn to the proof of (2.32). We remark that, for $u, v \in H_{1}$ and $\mu$ a.e. $x \in \Omega$, omitting for simplicity the argument $x$, applying the Cauchy-Schwarz inequality and (5.6),

$$
\frac{A(u, v-u)}{\varepsilon+a(u)} \leq \frac{a(u) a(v)-a(u)^{2}}{\varepsilon+a(u)} \leq \varepsilon+a(v)-a(u) .
$$

Using (2.31) where we replace $v$ by $v-\widehat{u}_{\varepsilon}$, we get

$$
\begin{aligned}
& \forall v \in \widehat{H}, \\
& \alpha\left\langle\widehat{u}_{\varepsilon}, v-\widehat{u}_{\varepsilon}\right\rangle+\mu(\Omega) \varepsilon+\psi(v)-\psi\left(\widehat{u}_{\varepsilon}\right) \geq\left\langle f, v-\widehat{u}_{\varepsilon}\right\rangle .
\end{aligned}
$$

Letting $v=\widehat{u}$ (the solution to (2.16)) in (2.40), we obtain

$$
\psi\left(\widehat{u}_{\varepsilon}\right)-\psi(\widehat{u}) \leq\left\langle\alpha \widehat{u}_{\varepsilon}-f, \widehat{u}-\widehat{u}_{\varepsilon}\right\rangle+\mu(\Omega) \varepsilon .
$$

Then, taking $v=\widehat{u}_{\varepsilon}$ in $(2.15)$ we get

$$
\psi(\widehat{u})-\psi\left(\widehat{u}_{\varepsilon}\right) \leq\left\langle\alpha \widehat{u}-f, \widehat{u}_{\varepsilon}-\widehat{u}\right\rangle .
$$


Adding the two inequalities, we then get (2.32). Let us now turn to the proof of (2.33). Setting $v=0$ in $(2.40)$, we get

$$
\alpha\left\|\widehat{u}_{\varepsilon}\right\|^{2}+\psi\left(\widehat{u}_{\varepsilon}\right) \leq \mu(\Omega) \varepsilon+\left\langle f, \widehat{u}_{\varepsilon}\right\rangle,
$$

which implies, since $\left\langle f, \widehat{u}_{\varepsilon}\right\rangle \leq \frac{1}{2 \alpha}\|f\|^{2}+\frac{\alpha}{2}\left\|\widehat{u}_{\varepsilon}\right\|^{2}$,

$$
\alpha^{2}\left\|\widehat{u}_{\varepsilon}\right\|^{2} \leq 2 \alpha \mu(\Omega) \varepsilon+\|f\|^{2} \leq(\|f\|+\sqrt{2 \alpha \mu(\Omega) \varepsilon})^{2} .
$$

This leads with (2.41) to

$$
\psi\left(\widehat{u}_{\varepsilon}\right)-\psi(\widehat{u}) \leq(2\|f\|+\sqrt{2 \alpha \mu(\Omega) \varepsilon})\left\|\widehat{u}_{\varepsilon}-\widehat{u}\right\|+\mu(\Omega) \varepsilon .
$$

Similarly, using (2.21) in (2.42) yields

$$
\psi(\widehat{u})-\psi\left(\widehat{u}_{\varepsilon}\right) \leq 2\|f\|\left\|\widehat{u}_{\varepsilon}-\widehat{u}\right\| .
$$

Using (2.32), we finally get (2.33).

The last step is to approximate the solution $\widehat{u}_{\varepsilon}$ to the regularized problem $(2.31)$, since it is nonlinear. For given initial $u^{(0)} \in \widehat{H}$ (and fixed $\varepsilon>0$ ), we now define the sequence $\left(u^{(k)}\right)_{k \in \mathbb{N}}$ by

$$
\begin{aligned}
& u^{(k+1)} \in \widehat{H}, \\
& \forall v \in \widehat{H}, \quad \alpha\left\langle u^{(k+1)}, v\right\rangle+\int_{\Omega} \frac{A\left(u^{(k+1)}, v\right)(x)}{\varepsilon+a\left(u^{(k)}\right)(x)} \mathrm{d} \mu=\langle f, v\rangle .
\end{aligned}
$$

We have the following result.

Lemma 2.14 Under Hypothesis 2.10, let $\alpha>0$ and $f \in H$. Let $\widehat{H}$ be a finite dimensional subspace of $H_{1}$, and let $u^{(0)} \in \widehat{H}$ and $\varepsilon>0$ be given. Then there exist a unique sequence $\left(u^{(k)}\right)_{k \in \mathbb{N}}$ defined by (2.43). Moreover, as $k \rightarrow \infty, u^{(k)}$ converges to $\widehat{u}_{\varepsilon}$, the unique solution to (2.31), and $\psi\left(u^{(k)}\right)$ converges to $\psi\left(\widehat{u}_{\varepsilon}\right)$.

Proof. The estimate

$$
\frac{\alpha}{2}\left\|u^{(k+1)}\right\|^{2}+\int_{\Omega} \frac{a\left(u^{(k+1)}\right)(x)^{2}}{\varepsilon+a\left(u^{(k)}\right)(x)} \mathrm{d} \mu \leq \frac{1}{2 \alpha}\|f\|^{2},
$$

obtained by letting $v=u^{(k+1)}$ in (2.43) and applying the Young inequality, shows that with a null right-hand side, the square linear system to be solved has the unique solution 0 . Hence it is invertible, showing the existence and uniqueness of the sequence $\left(u^{(k)}\right)_{k \in \mathbb{N}}$.

We then let $v=u^{(k+1)}-u^{(k)}$ in (2.43). Since the Cauchy-Schwarz inequality implies, $\mu$ a.e. in $\Omega$,

$$
\frac{a\left(u^{(k+1)}\right)}{\varepsilon+a\left(u^{(k)}\right)}\left(a\left(u^{(k+1)}\right)-a\left(u^{(k)}\right)\right) \leq \frac{A\left(u^{(k+1)}, u^{(k+1)}-u^{(k)}\right)}{\varepsilon+a\left(u^{(k)}\right)},
$$

we get from (5.4) proved in Lemma 5.1 that

$$
\begin{aligned}
& \frac{\alpha}{2}\left(\left\|u^{(k+1)}\right\|^{2}+\left\|u^{(k+1)}-u^{(k)}\right\|^{2}-\left\|u^{(k)}\right\|^{2}\right) \\
& +\int_{\Omega}\left(F_{\varepsilon}\left(a\left(u^{(k+1)}\right)(x)\right)-F_{\varepsilon}\left(a\left(u^{(k)}\right)(x)\right)+\frac{\left(a\left(u^{(k+1)}\right)(x)-a\left(u^{(k)}\right)(x)\right)^{2}}{2\left(\varepsilon+a\left(u^{(k)}\right)(x)\right)}\right) \mathrm{d} \mu \\
& \leq\left\langle f, u^{(k+1)}-u^{(k)}\right\rangle .
\end{aligned}
$$


Therefore, summing the above inequality for $k=0, \ldots, m$ and applying the Young inequality to the right-hand side, we get

$$
\begin{aligned}
& \frac{\alpha}{2}\left(\left\|u^{(m+1)}\right\|^{2}+\sum_{k=0}^{m}\left\|u^{(k+1)}-u^{(k)}\right\|^{2}-\left\|u^{(0)}\right\|^{2}\right) \\
& +\int_{\Omega}\left(F_{\varepsilon}\left(a\left(u^{(m+1)}\right)(x)\right)-F_{\varepsilon}\left(a\left(u^{(0)}\right)(x)\right)\right. \\
& \left.+\frac{1}{2} \sum_{k=0}^{m} \frac{\left(a\left(u^{(k+1)}\right)(x)-a\left(u^{(k)}\right)(x)\right)^{2}}{\varepsilon+a\left(u^{(k)}\right)(x)}\right) \mathrm{d} \mu \\
& \leq \frac{1}{\alpha}\|f\|^{2}+\frac{\alpha}{4}\left\|u^{(m+1)}\right\|^{2}-\left\langle f, u^{(0)}\right\rangle .
\end{aligned}
$$

This proves on one hand that $\frac{\alpha}{4}\left\|u^{(m+1)}\right\|^{2}+\int_{\Omega} F_{\varepsilon}\left(a\left(u^{(m+1)}\right)(x)\right) \mathrm{d} \mu$ remains bounded independently of $m$, and using (5.3) proved in Lemma 5.1, we get that $\psi\left(u^{(m+1)}\right)$ remains bounded.

This proves on the other hand that the two series in the left-hand side of the above inequality converge, and therefore that

$$
\left\|u^{(k+1)}-u^{(k)}\right\| \rightarrow 0, \quad \text { as } k \rightarrow \infty .
$$

We next observe that $v \mapsto\|a(v)\|_{L_{\mu}^{2}(\Omega)}$ is a semi-norm on $H_{1}$. Since $\widehat{H}$ has finite dimension, this implies that there exist a constant $\widehat{M}$ such that

$$
\forall v \in \widehat{H}, \quad\|a(v)\|_{L_{\mu}^{2}(\Omega)} \leq \widehat{M}\|v\| .
$$

Writing

$$
\left\|a\left(u^{(k+1)}\right)-a\left(u^{(k)}\right)\right\|_{L_{\mu}^{2}(\Omega)} \leq\left\|a\left(u^{(k+1)}-u^{(k)}\right)\right\|_{L_{\mu}^{2}(\Omega)} \leq \widehat{M}\left\|u^{(k+1)}-u^{(k)}\right\|,
$$

we get that $a\left(u^{(k+1)}\right)-a\left(u^{(k)}\right) \rightarrow 0$ in $L_{\mu}^{2}(\Omega)$. Using again that the dimension of $\widehat{H}$ is finite, we deduce from (2.44) that there exists a subsequence of $\left(u^{(k)}\right)_{k \in \mathbb{N}}$, again denoted $\left(u^{(k)}\right)_{k \in \mathbb{N}}$, strongly convergent in the finite dimensional vector space to some element $u \in \widehat{H}$. This implies that $\left(a\left(u^{(k)}\right)\right)_{k \in \mathbb{N}}$ converges in $L_{\mu}^{2}(\Omega)$ to $a(u)$, and that $\psi\left(u^{(k)}\right)$ tends to $\psi(u)$. Using (2.47), we may then pass to the limit in (2.43) for this extracted subsequence, and we get that the limit $u$ satisfies (2.31). Since the solution to (2.31) is unique, we get that the whole sequence $\left(u^{(k)}\right)_{k \in \mathbb{N}}$ converges to this solution.

Lemma 2.15 With the assumptions of Lemma 2.14, assume further that

$$
\forall v \in \widehat{H},\|a(v)\|_{L_{\mu}^{\infty}(\Omega)}<\infty .
$$

Then the convergence of $u^{(k)}$ to $\widehat{u}_{\varepsilon}$ as $k \rightarrow \infty$ is asymptotically geometric with ratio arbitrarily close to

$$
\frac{\left\|a\left(\widehat{u}_{\varepsilon}\right)\right\|_{L_{\mu}^{\infty}(\Omega)}}{\varepsilon+\left\|a\left(\widehat{u}_{\varepsilon}\right)\right\|_{L_{\mu}^{\infty}(\Omega)}}<1
$$


Proof. With the assumption (2.50), $v \mapsto\|a(v)\|_{L_{\mu}^{\infty}(\Omega)}$ is a semi-norm on $\widehat{H}$, which is finite dimensional. Thus there exists a constant $\widehat{G}$ such that

$$
\forall v \in \widehat{H}, \quad\|a(v)\|_{L_{\mu}^{\infty}(\Omega)} \leq \widehat{G}\|v\| .
$$

It implies that $a\left(u^{(k)}\right) \rightarrow a\left(\widehat{u}_{\varepsilon}\right)$ in $L_{\mu}^{\infty}(\Omega)$. Then, take successively $v=u^{(k+2)}-u^{(k+1)}$ in $(2.43)$, and in (2.43) with $k$ incremented of 1 . The difference yields

$$
\begin{gathered}
\alpha\left\|u^{(k+2)}-u^{(k+1)}\right\|^{2}+\int_{\Omega} \frac{A\left(u^{(k+2)}, u^{(k+2)}-u^{(k+1)}\right)(x)}{\varepsilon+a\left(u^{(k+1)}\right)(x)} \mathrm{d} \mu \\
-\int_{\Omega} \frac{A\left(u^{(k+1)}, u^{(k+2)}-u^{(k+1)}\right)(x)}{\varepsilon+a\left(u^{(k)}\right)(x)} \mathrm{d} \mu=0 .
\end{gathered}
$$

We deduce omitting the $x$ that

$$
\begin{aligned}
& \alpha\left\|u^{(k+2)}-u^{(k+1)}\right\|^{2}+\int_{\Omega} \frac{a\left(u^{(k+2)}-u^{(k+1)}\right)^{2}}{\varepsilon+a\left(u^{(k+1)}\right)} \mathrm{d} \mu \\
= & \int_{\Omega} A\left(u^{(k+1)}, u^{(k+2)}-u^{(k+1)}\right)\left(\frac{1}{\varepsilon+a\left(u^{(k)}\right)}-\frac{1}{\varepsilon+a\left(u^{(k+1)}\right)}\right) \mathrm{d} \mu \\
\leq & \int_{\Omega} a\left(u^{(k+1)}\right) a\left(u^{(k+2)}-u^{(k+1)}\right) \frac{\left|a\left(u^{(k+1)}\right)-a\left(u^{(k)}\right)\right|}{\left(\varepsilon+a\left(u^{(k)}\right)\right)\left(\varepsilon+a\left(u^{(k+1)}\right)\right)} \mathrm{d} \mu \\
\leq & \frac{1}{2} \int_{\Omega}\left(\frac{a\left(u^{(k+2)}-u^{(k+1)}\right)^{2}}{\varepsilon+a\left(u^{(k+1)}\right)}+a\left(u^{(k+1)}\right)^{2} \frac{\left|a\left(u^{(k+1)}\right)-a\left(u^{(k)}\right)\right|^{2}}{\left(\varepsilon+a\left(u^{(k)}\right)\right)^{2}\left(\varepsilon+a\left(u^{(k+1)}\right)\right)}\right) \mathrm{d} \mu,
\end{aligned}
$$

and therefore that

$$
\begin{aligned}
& 2 \alpha\left\|u^{(k+2)}-u^{(k+1)}\right\|^{2}+\int_{\Omega} \frac{a\left(u^{(k+2)}-u^{(k+1)}\right)^{2}}{\varepsilon+a\left(u^{(k+1)}\right)} \mathrm{d} \mu \\
\leq & \int_{\Omega} a\left(u^{(k+1)}\right)^{2} \frac{a\left(u^{(k+1)}-u^{(k)}\right)^{2}}{\left(\varepsilon+a\left(u^{(k)}\right)\right)^{2}\left(\varepsilon+a\left(u^{(k+1)}\right)\right)} \mathrm{d} \mu .
\end{aligned}
$$

Now, since $a\left(u^{(k)}\right) \rightarrow a\left(\widehat{u}_{\varepsilon}\right)$ in $L_{\mu}^{\infty}(\Omega)$, for given $\eta>0$ there exist some $k_{0}$ such that

$$
\forall k \geq k_{0}, \mu \text { a.e. in } \Omega, \quad \frac{a\left(u^{(k+1)}\right)^{2}}{\left(\varepsilon+a\left(u^{(k)}\right)\right)\left(\varepsilon+a\left(u^{(k+1)}\right)\right)} \leq(r+\eta)^{2},
$$

where $r$ is the left-hand side of (2.51). Then, for $k \geq k_{0}$ one has

$$
\begin{aligned}
& 2 \alpha\left\|u^{(k+2)}-u^{(k+1)}\right\|^{2}+\int_{\Omega} \frac{a\left(u^{(k+2)}-u^{(k+1)}\right)^{2}}{\varepsilon+a\left(u^{(k+1)}\right)} \mathrm{d} \mu \\
\leq & (r+\eta)^{2} \int_{\Omega} \frac{a\left(u^{(k+1)}-u^{(k)}\right)^{2}}{\varepsilon+a\left(u^{(k)}\right)} \mathrm{d} \mu,
\end{aligned}
$$

which implies that for $k \geq k_{0}$

$$
\int_{\Omega} \frac{a\left(u^{(k+1)}-u^{(k)}\right)^{2}}{\varepsilon+a\left(u^{(k)}\right)} \mathrm{d} \mu \leq(r+\eta)^{2\left(k-k_{0}\right)} \int_{\Omega} \frac{a\left(u^{\left(k_{0}+1\right)}-u^{\left(k_{0}\right)}\right)^{2}}{\varepsilon+a\left(u^{\left(k_{0}\right)}\right)} \mathrm{d} \mu .
$$


Plugging this in (2.57) we deduce that for $k \geq k_{0}$,

$$
\left\|u^{(k+2)}-u^{(k+1)}\right\| \leq C(r+\eta)^{k-k_{0}},
$$

where $C$ does not depend on $k$. If $r+\eta<1$ we finally write for $k \geq k_{0}$

$$
\left\|u^{(k)}-\widehat{u}_{\varepsilon}\right\| \leq \sum_{k^{\prime}=k}^{\infty}\left\|u^{\left(k^{\prime}+1\right)}-u^{\left(k^{\prime}\right)}\right\| \leq C(r+\eta)^{k-k_{0}},
$$

which proves the claim.

\section{Transient problem}

\subsection{Continuous framework}

Assuming Hypothesis 2.6 (we again notice that the assumptions $\psi \geq 0$ and $\psi(0)=0$ bring no restriction to generality in Problem (3.3) below), let $T>0$ be given. The space $L^{2}(0, T ; H)$ is defined as the Hilbert space of all measurable, almost everywhere defined functions $u$ (in the so-called "Bochner integral" sense, recalled for example in [11]) from $(0, T)$ to $H$ such that $\|u\|_{L^{2}(0, T ; H)}^{2}:=\int_{0}^{T}\|u(t)\|^{2} \mathrm{~d} t<\infty$. The set $B_{T}$ is defined as

$$
B_{T}=\left\{u \in L^{2}(0, T ; H) ; \int_{0}^{T} \psi(u(t)) \mathrm{d} t<\infty\right\} .
$$

For any $u \in L_{\text {loc }}^{1}(0, T ; H)$, we denote by $\partial_{t} u \in L_{\text {loc }}^{1}(0, T ; H)$ the time weak derivative of $u$ when it exists, that is $\partial_{t} u \in L_{\text {loc }}^{1}(0, T ; H)$ is such that

$$
\forall \varphi \in C_{c}^{1}(] 0, T[), \int_{0}^{T} \varphi^{\prime}(t) u(t) \mathrm{d} t=-\int_{0}^{T} \varphi(t) \partial_{t} u(t) \mathrm{d} t .
$$

We recall that $C^{\infty}([0, T] ; H)$ is dense in the Hilbert spaces $L^{2}(0, T ; H)$ and in $H^{1}(0, T ; H):=$ $\left\{u \in L^{2}(0, T ; H) ; \partial_{t} u \in L^{2}(0, T ; H)\right\}$, and that $H^{1}(0, T ; H) \subset C^{0}([0, T] ; H)$ (see [11]). We also recall that, for all $u, v \in H^{1}(0, T ; H)$, we have for any $t_{1}, t_{2} \in[0, T]$

$$
\int_{t_{1}}^{t_{2}}\left\langle\partial_{t} u(t), v(t)\right\rangle \mathrm{d} t+\int_{t_{1}}^{t_{2}}\left\langle u(t), \partial_{t} v(t)\right\rangle \mathrm{d} t=\left\langle u\left(t_{2}\right), v\left(t_{2}\right)\right\rangle-\left\langle u\left(t_{1}\right), v\left(t_{1}\right)\right\rangle .
$$

Let $f \in L^{2}(0, T ; H)$ and $u^{0} \in B$ be given. We look in this section for a function $u$ such that

$$
\begin{aligned}
& u \in H^{1}(0, T ; H) \cap B_{T}, \\
& u(0)=u^{0}, \\
& \quad \int_{0}^{T}\left\langle\partial_{t} u(t), v(t)-u(t)\right\rangle \mathrm{d} t+\int_{0}^{T}(\psi(v(t))-\psi(u(t))) \mathrm{d} t \\
& \geq \int_{0}^{T}\langle f(t), v(t)-u(t)\rangle \mathrm{d} t, \quad \forall v \in B_{T} .
\end{aligned}
$$


Let us remark that the inequality (3.3) implies

$$
\begin{aligned}
& \int_{t_{1}}^{t_{2}}\left\langle\partial_{t} u(t), v(t)-u(t)\right\rangle \mathrm{d} t+\int_{t_{1}}^{t_{2}}(\psi(v(t))-\psi(u(t))) \mathrm{d} t \\
\geq & \int_{t_{1}}^{t_{2}}\langle f(t), v(t)-u(t)\rangle \mathrm{d} t, \quad \forall v \in B_{t_{1}, t_{2}}, \forall t_{1}<t_{2} \in[0, T],
\end{aligned}
$$

where $B_{t_{1}, t_{2}}$ denotes the set of all $v \in L^{2}\left(t_{1}, t_{2} ; H\right)$ such that $\int_{t_{1}}^{t_{2}} \psi(v(t)) \mathrm{d} t<\infty$. It indeed suffices, for a given $\tilde{v} \in B_{t_{1}, t_{2}}$, to define $v(t)=\tilde{v}(t)$ for a.e. $\left.t \in\right] t_{1}, t_{2}[$ and $v(t)=u(t)$ for a.e. $t \in] 0, t_{1}[\cup] t_{2}, T$, and then use this $v \in B_{T}$ as test function in (3.3). Then, since the restriction of $v \in B_{T}$ to any interval ] $t_{1}, t_{2}$ [ belongs to $B_{t_{1}, t_{2}}$, we conclude that the inequality (3.3) is equivalent to

$$
\begin{aligned}
& \left\langle\partial_{t} u(t), v(t)-u(t)\right\rangle+\psi(v(t))-\psi(u(t)) \\
\geq & \langle f(t), v(t)-u(t)\rangle, \text { for a.e. } t \in] 0, T\left[\quad \forall v \in B_{T} .\right.
\end{aligned}
$$

One can derive even a stronger formulation. Let $E \subset] 0, T[$ a set such that meas (] $0, T\lceil\backslash E)=0$ and for all $t \in E$, as $t_{1}, t_{2} \rightarrow t$ with $0<t_{1}<t<t_{2}<T$,

$$
\begin{gathered}
f_{t_{1}}^{t_{2}} \psi(u(s)) d s \rightarrow \psi(u(t)), \\
f_{t_{1}}^{t_{2}}\left\langle\partial_{t} u(s)-f(s), u(s)\right\rangle d s \rightarrow\left\langle\partial_{t} u(t)-f(t), u(t)\right\rangle, \\
f_{t_{1}}^{t_{2}}\left(\partial_{t} u(s)-f(s)\right) d s \rightarrow \partial_{t} u(t)-f(t) \text { weakly in } H,
\end{gathered}
$$

where the bar integral denotes the average, i.e. the integral normalized by the volume. Dividing (3.4) by $t_{2}-t_{1}$, passing to the limit as $t_{1}, t_{2} \rightarrow t$ for all $t \in E$ and taking $v$ equal to a constant element of $B$, we get

$$
\begin{aligned}
& \left\langle\partial_{t} u(t), v-u(t)\right\rangle+\psi(v)-\psi(u(t)) \\
\geq & \langle f(t), v-u(t)\rangle, \forall v \in B, \quad \text { for a.e. } t \in] 0, T[.
\end{aligned}
$$

This strong formulation is therefore equivalent to (3.5) and to (3.3). It may also be written as

$$
\left.\partial_{t} u(t)+\partial \psi(u(t)) \ni f(t) \quad \text { for a.e. } t \in\right] 0, T[,
$$

with the subdifferential defined in (2.8). However, in this paper we shall not use this formulation.

The study of existence and uniqueness for Problem (3.3) is given in [22, Theorem 20], following [6], in the case when $f=0$, using the semi-group approach and the Yosida regularization. Since we focus on the approximation of this problem, we indeed recover the existence through the convergence of a semi-discrete (in time) approximation. A convergence result is however established for general time-space approximations.

The following lemma is classical. We recall its short proof.

Lemma 3.1 Under Hypothesis 2.6, let $T>0$ be given, and $f \in L^{2}(0, T ; H)$. If $u_{1}$ and $u_{2}$ are two solutions to Problem (3.3), with possibly different initial data, then $\left\|u_{2}(t)-u_{1}(t)\right\|$ is nonincreasing in $[0, T]$. In particular, there exists at most one solution to Problem (3.3) with a given initial data $u^{0} \in B$. 
Proof. Let $u_{1}$ and $u_{2}$ be two solutions to Problem (3.3), with possibly different intial data. Choosing, for given $t_{1}<t_{2} \in[0, T], v=u_{2}$ (respectively $v=u_{1}$ ) in (3.4) with $u=u_{1}$ (respectively $u=u_{2}$ ), and adding the two obtained inequalities, we get

$$
\int_{t_{1}}^{t_{2}}\left\langle\partial_{t} u_{1}(t)-\partial_{t} u_{2}(t), u_{2}(t)-u_{1}(t)\right\rangle \mathrm{d} t \geq 0
$$

Taking into account (3.2), it yields

$$
\frac{1}{2}\left\|u_{2}\left(t_{2}\right)-u_{1}\left(t_{2}\right)\right\|^{2} \leq \frac{1}{2}\left\|u_{2}\left(t_{1}\right)-u_{1}\left(t_{1}\right)\right\|^{2},
$$

which proves the claim.

Lemma 3.2 Under Hypothesis 2.6, let $T>0$ be given, $f \in L^{2}(0, T ; H)$ and $u^{0} \in B$. Then any solution u to Problem (3.3) verifies the a priori estimates

$$
\begin{gathered}
\|u(t)\| \leq\left\|u^{0}\right\|+2 \sqrt{t}\|f\|_{L^{2}(0, T ; H)}, \quad \text { for all } t \in[0, T], \\
\int_{0}^{T} \psi(u(t)) \mathrm{d} t \leq \frac{1}{2}\left(\left\|u^{0}\right\|+2 \sqrt{T}\|f\|_{L^{2}(0, T ; H)}\right)^{2} .
\end{gathered}
$$

Proof. We take $v=0$ in (3.4), which gives for all $t_{1}<t_{2} \in[0, T]$

$$
\frac{1}{2}\left\|u\left(t_{2}\right)\right\|^{2}+\int_{t_{1}}^{t_{2}} \psi(u(t)) \mathrm{d} t \leq \frac{1}{2}\left\|u\left(t_{1}\right)\right\|^{2}+\int_{t_{1}}^{t_{2}}\langle f(t), u(t)\rangle \mathrm{d} t .
$$

In particular this implies by taking $t_{1}=0$ that for all $t \in[0, T]$,

$$
\|u(t)\|^{2} \leq\left\|u^{0}\right\|^{2}+2\|f\|_{L^{2}(0, T ; H)}\left(\int_{0}^{t}\|u(s)\|^{2} \mathrm{~d} s\right)^{1 / 2} .
$$

Defining $\varphi(t)=\int_{0}^{t}\|u(s)\|^{2} \mathrm{~d} s$, it satisfies the differential inequality $\varphi^{\prime}(t) \leq\left\|u^{0}\right\|^{2}+2\|f\| \sqrt{\varphi(t)}$, which implies that

$$
\frac{d}{d t}\left(\frac{\varphi(t)}{\left\|u^{0}\right\|^{2}+2\|f\| \sqrt{\varphi(t)}}\right) \leq 1 .
$$

Taking into account that $\varphi(0)=0$, we deduce that

$$
\frac{\varphi(t)}{\left\|u^{0}\right\|^{2}+2\|f\| \sqrt{\varphi(t)}} \leq t,
$$

i.e.

$$
\varphi(t)-2 t\|f\| \sqrt{\varphi(t)}-t\left\|u^{0}\right\|^{2} \leq 0,
$$

which yields

$$
\sqrt{\varphi(t)} \leq t\|f\|+\left(t^{2}\|f\|^{2}+t\left\|u^{0}\right\|^{2}\right)^{1 / 2} .
$$


Plugging this into (3.11) we obtain

$$
\begin{aligned}
\|u(t)\|^{2} & \leq\left\|u^{0}\right\|^{2}+2\|f\|\left(t\|f\|+\left(t^{2}\|f\|^{2}+t\left\|u^{0}\right\|^{2}\right)^{1 / 2}\right) \\
& \leq\left\|u^{0}\right\|^{2}+4 t\|f\|^{2}+2 \sqrt{t}\left\|u^{0}\right\|\|f\| \\
& \leq\left(\left\|u^{0}\right\|+2 \sqrt{t}\|f\|\right)^{2}
\end{aligned}
$$

which proves (3.8). Finally, coming back to (3.10) we easily get (3.9).

Remark 3.3 A generalization of the previous result is as follows. Under Hypothesis 2.6, let $T>0$ be given, $f_{1}, f_{2} \in L^{2}(0, T ; H)$ and $u_{1}^{0}, u_{2}^{0} \in B$. Then two solutions $u_{1}$, $u_{2}$ to the Problem (3.3) with respective data verify

$$
\left\|u_{2}(t)-u_{1}(t)\right\| \leq\left\|u_{2}^{0}-u_{1}^{0}\right\|+2 \sqrt{t}\left\|f_{2}-f_{1}\right\|_{L^{2}(0, T ; H)}, \quad \forall t \in[0, T] .
$$

This is obtained by taking as test function $v=u_{2}$ for the formulation associated to $u_{1}$, taking $v=u_{1}$ as test function for the formulation associated to $u_{2}$, adding the results and arguing as above by a Gronwall lemma.

Remark 3.4 The lemmas 3.1 and 3.2 indeed only use that $u^{0} \in H$, and not that $u^{0} \in B$. However, in order to get an estimate on $\partial_{t} u$, the property $\psi\left(u^{0}\right)<\infty$ is needed, as we shall see below.

\subsection{Time-space implicit approximation}

In this subsection we consider the space approximation (2.15), but applied to the transient problem of the previous subsection. We thus consider the following approximate method. Assuming Hypothesis 2.6, let $\widehat{H}$ be a closed subspace of $H$, and let $\widehat{B}=\widehat{H} \cap B$. We first approximate $u^{0} \in B$ by some

$$
\widehat{u}^{0} \in \widehat{B} \text {, satisfying } \psi\left(\widehat{u}^{0}\right) \leq G, \psi\left(u^{0}\right) \leq G,
$$

for some constant $G \geq 0$. We then take $n \in \mathbb{N}^{\star}$, we define the timestep $\tau=T / n$, the sequence $\left(f^{k}\right)_{k=1, \ldots, n}$ of elements of $H$ and the function $f_{n}(t)$ by

$$
\begin{aligned}
& f^{k}=\frac{1}{\tau} \int_{(k-1) \tau}^{k \tau} f(t) \mathrm{d} t, \forall k=1, \ldots, n, \\
& \left.f_{n}(t)=f^{k}, \text { for a.e. } t \in\right](k-1) \tau, k \tau[, \forall k=1, \ldots, n .
\end{aligned}
$$

The sequence $\left(\widehat{u}^{k}\right)_{k=1, \ldots, n}$ is then defined by

$$
\begin{aligned}
& \widehat{u}^{k} \in \widehat{B} \\
& \left\langle D^{k} \widehat{u}, w-\widehat{u}^{k}\right\rangle+\psi(w)-\psi\left(\widehat{u}^{k}\right) \geq\left\langle f^{k}, w-\widehat{u}^{k}\right\rangle, \forall w \in \widehat{B}, \forall k=1, \ldots, n,
\end{aligned}
$$

where $\left(D^{k} \widehat{u}\right)_{k=1, \ldots, n}$ is expressed in terms of $\widehat{u}^{k}$ and $\widehat{u}^{k-1}$ by

$$
D^{k} \widehat{u}=\frac{\widehat{u}^{k}-\widehat{u}^{k-1}}{\tau}, k=1, \ldots, n .
$$


The existence and uniqueness of $\left(\widehat{u}^{k}\right)_{k=1, \ldots, n}$ solution to (3.16) is obvious since this problem has the same form as (2.15) with $\alpha=1 / \tau$ and $f=f^{k}+\widehat{u}^{k-1} / \tau$. Let us denote by

$$
\begin{aligned}
& \left.\left.\widehat{u}(t)=\widehat{u}^{k} \text { and } D_{t} \widehat{u}(t)=D^{k} \widehat{u}, \forall t \in\right](k-1) \tau, k \tau\right], \forall k=1, \ldots, n, \\
& \widehat{u}(0)=\widehat{u}^{0},
\end{aligned}
$$

hence $\widehat{u}(t)$ is defined for all $t \in[0, T]$. We have the following estimates on $\widehat{u}$ and $D_{t} \widehat{u}$.

Lemma 3.5 Under Hypothesis 2.6, let $T>0, f \in L^{2}(0, T ; H)$ and $u^{0} \in B$ be given. Let $\widehat{H}$ be a closed subspace of $H$, and let $\widehat{B}=\widehat{H} \cap B$. Let $\widehat{u}^{0} \in \widehat{B}$ be such that (3.14) holds. Let $n \in \mathbb{N}^{\star}$, $\tau=T / n$, and let $\widehat{u}$ and $D_{t} \widehat{u}$ be defined by (3.15)-(3.18). Then, for $C_{2}$ given by (3.25), there holds

$$
\begin{gathered}
\psi(\widehat{u}(t)) \leq C_{2} / 2, \forall t \in[0, T], \\
\left\|D_{t} \widehat{u}\right\|_{L^{2}(0, T ; H)}^{2} \leq C_{2}, \\
\|\widehat{u}(t)\| \leq\left\|\widehat{u}^{0}\right\|+\left(T C_{2}\right)^{1 / 2}, \forall t \in[0, T],
\end{gathered}
$$

and

$$
\left\|\widehat{u}\left(t_{2}\right)-\widehat{u}\left(t_{1}\right)\right\| \leq\left(C_{2}\right)^{1 / 2}\left(\left|t_{2}-t_{1}\right|+\tau\right)^{1 / 2}, \forall t_{1}, t_{2} \in[0, T] .
$$

Proof. Let us first remark that, according to the Cauchy-Schwarz inequality,

$$
\left\|f^{k}\right\|^{2} \leq \frac{1}{\tau} \int_{(k-1) \tau}^{k \tau}\|f(t)\|^{2} \mathrm{~d} t
$$

which leads to

$$
\sum_{k=1}^{n} \tau\left\|f^{k}\right\|^{2} \leq\|f\|_{L^{2}(0, T ; H)}^{2}
$$

Setting $w=\widehat{u}^{k-1}$ in (3.16), we get

$$
\tau\left\|D^{k} \widehat{u}\right\|^{2}+\psi\left(\widehat{u}^{k}\right)-\psi\left(\widehat{u}^{k-1}\right) \leq\left\langle f^{k}, \widehat{u}^{k}-\widehat{u}^{k-1}\right\rangle \leq \frac{\tau}{2}\left\|f^{k}\right\|^{2}+\frac{\tau}{2}\left\|D^{k} \widehat{u}\right\|^{2},
$$

which gives, summing for $k=1, \ldots, m$ for a given $m=1, \ldots, n$,

$$
\begin{aligned}
\psi\left(\widehat{u}^{m}\right)+\frac{1}{2}\left\|D_{t} \widehat{u}\right\|_{L^{2}(0, m \tau ; H)}^{2} & \leq \psi\left(\widehat{u}^{0}\right)+\frac{1}{2}\|f\|_{L^{2}(0, T ; H)}^{2} \\
& \leq G+\frac{1}{2}\|f\|_{L^{2}(0, T ; H)}^{2} .
\end{aligned}
$$

This gives (3.19) and (3.20) with

$$
C_{2}=2 G+\|f\|_{L^{2}(0, T ; H)}^{2} .
$$

We then write

$$
\widehat{u}^{m}=\widehat{u}^{0}+\sum_{k=1}^{m} \tau D^{k} \widehat{u}, \forall m=1, \ldots, n,
$$

which implies, using the Cauchy-Schwarz inequality and (3.20)

$$
\left\|\widehat{u}^{m}-\widehat{u}^{0}\right\| \leq \sum_{k=1}^{m} \tau\left\|D^{k} \widehat{u}\right\| \leq\left(m \tau\left\|D_{t} \widehat{u}\right\|_{L^{2}(0, m \tau ; H)}^{2}\right)^{1 / 2} \leq\left(T C_{2}\right)^{1 / 2} .
$$


This leads to (3.21). Finally, let $t_{1}<t_{2} \in[0, T]$ and $k_{1}, k_{2}=0, \ldots, n$ such that $\left(k_{1}-1\right) \tau<t_{1} \leq$ $k_{1} \tau$ and $\left(k_{2}-1\right) \tau<t_{2} \leq k_{2} \tau$, which implies that $\left(k_{2}-1-k_{1}\right) \tau<t_{2}-t_{1}$. We have

$$
\left\|\widehat{u}\left(t_{2}\right)-\widehat{u}\left(t_{1}\right)\right\| \leq \sum_{k=k_{1}+1}^{k_{2}} \tau\left\|D^{k} \widehat{u}\right\| \leq\left(\left(k_{2}-k_{1}\right) \tau \sum_{k=k_{1}+1}^{k_{2}} \tau\left\|D^{k} \widehat{u}\right\|^{2}\right)^{1 / 2},
$$

which provides (3.22), using (3.20).

We may now prove the following convergence result.

Theorem 3.6 Under Hypothesis 2.6, let $T>0, f \in L^{2}(0, T ; H)$ and $u^{0} \in B$ be given. Let $\left(\widehat{H}_{n}\right)_{n \in \mathbb{N}^{\star}}$ be a sequence of closed subspaces of $H$, and let $\widehat{B}_{n}=\widehat{H}_{n} \cap B$. We assume that

$$
\lim _{n \rightarrow \infty} \inf _{w \in \widehat{B}_{n}}\left(\|w-v\|+(\psi(w)-\psi(v))^{+}\right)=0, \forall v \in B .
$$

For all $n \in \mathbb{N}^{\star}$, let $\widehat{u}_{n}^{0}$ satisfy

$$
\widehat{u}_{n}^{0} \in \widehat{B}_{n}, \quad\left\|\widehat{u}_{n}^{0}-u^{0}\right\| \rightarrow 0 \text { as } n \rightarrow \infty, \quad G:=\sup _{n} \psi\left(\widehat{u}_{n}^{0}\right)<\infty,
$$

which is possible according to (3.27). Let $\tau=T / n$, let $\widehat{u}_{n}$ and $D_{t} \widehat{u}_{n}$ be defined by (3.15)-(3.18) where $\widehat{H}$ has to be replaced by $\widehat{H}_{n}$ and $\widehat{u}^{0}=\widehat{u}_{n}^{0}$. Then $\widehat{u}_{n}(t)$ weakly converges in $H$ to $u(t)$ as $n \rightarrow \infty$, uniformly with respect to $t \in[0, T]$, and $u$ is solution to Problem (3.3). Moreover, $u$ satisfies

$$
\begin{gathered}
\psi(u(t)) \leq \frac{1}{2} C_{2}, \forall t \in[0, T], \\
\left\|\partial_{t} u\right\|_{L^{2}(0, T ; H)}^{2} \leq C_{2}, \\
\left\|u\left(t_{2}\right)-u\left(t_{1}\right)\right\| \leq\left(C_{2}\right)^{1 / 2}\left|t_{2}-t_{1}\right|^{1 / 2}, \forall t_{1}, t_{2} \in[0, T],
\end{gathered}
$$

where $C_{2}=2 G+\|f\|_{L^{2}(0, T ; H)}^{2}$.

Remark 3.7 Letting $\widehat{H}^{n}=H$ and $\widehat{u}_{n}^{0}=u^{0}$ for all $n \in \mathbb{N}^{\star}$ (semi-discretization in time), Theorem 3.6 provides the existence of a solution to Problem (3.3), and allows for taking $G=$ $\psi\left(u^{0}\right)$ and $C_{2}=2 \psi\left(u^{0}\right)+\|f\|_{L^{2}(0, T ; H)}^{2}$ in (3.29)-(3.31), since the solution is unique.

Remark 3.8 Theorem 3.11 shows indeed stronger convergence properties.

Proof of Theorem 3.6. Applying Lemma 3.5, we get that the hypotheses of the Ascolitype Lemma 5.3 (provided in appendix) are fulfilled, from which we deduce that there exists $u \in C^{0}([0, T] ; H)$ and of a subsequence of $\left(\widehat{u}_{n}\right)_{n \in \mathbb{N}^{*}}$, again denoted $\left(\widehat{u}_{n}\right)_{n \in \mathbb{N}^{*}}$, such that $\widehat{u}_{n}(t)$ converges to $u(t)$ weakly in $H$, uniformly for $t \in[0, T]$. Note that, according to (3.28), we have $u(0)=u^{0}$. Using (3.19) and the lower semi-continuity of $\psi$, we get that (3.29) holds, and in particular that $u(t) \in B$ and $u \in B_{T}$. Next, (3.31) comes directly from (5.10). Then, according to (3.20), we have that $\left(D_{t} \widehat{u}_{n}\right)_{n \in \mathbb{N}}$ is bounded in the Hilbert space $L^{2}(0, T ; H)$. Hence extracting a subsequence, $D_{t} \widehat{u}_{n}$ weakly converges in $L^{2}(0, T ; H)$ to some $w \in L^{2}(0, T ; H)$ satisfying $\|w\|_{L^{2}(0, T ; H)}^{2} \leq C_{2}$. We then have, for a given $\varphi \in C_{c}^{1}(] 0, T[)$,

$$
-\int_{0}^{T} \widehat{u}_{n}(t) \varphi^{\prime}(t) \mathrm{d} t=-\sum_{k=1}^{n} \widehat{u}^{k} \int_{(k-1) \tau}^{k \tau} \varphi^{\prime}(t) \mathrm{d} t=\sum_{k=1}^{n} \tau D^{k} \widehat{u} \varphi((k-1) \tau) .
$$


Passing to the limit weakly in $H$ in the above relation gives

$$
-\int_{0}^{T} u(t) \varphi^{\prime}(t) \mathrm{d} t=\int_{0}^{T} w(t) \varphi(t) \mathrm{d} t
$$

which shows that $\partial_{t} u=w \in L^{2}$. This concludes the proof that $u \in H^{1}(0, T ; H)$ with the estimate (3.30).

Let us finally prove that this function $u$ verifies (3.3). Take $v \in B_{T}$, and for $n \in \mathbb{N}^{\star}$, consider

$$
\left.v_{n}(t)=\underset{w \in \widehat{B}_{n}}{\operatorname{argmin}}\left(\|w-v(t)\|^{2}+(\psi(w)-\psi(v(t)))^{+}\right) \text {, for a.e. } t \in\right] 0, T[.
$$

Indeed, the existence of $v_{n}(t)$ is given by Lemma 2.7, for the lower semi-continuous function $w \mapsto(\psi(w)-\psi(v(t)))^{+}$. Since $0 \in \widehat{B}_{n}, v_{n}(t)$ verifies

$$
\left.\left\|v_{n}(t)-v(t)\right\|^{2}+\left(\psi\left(v_{n}(t)\right)-\psi(v(t))\right)^{+} \leq\|v(t)\|^{2} \text {, for a.e. } t \in\right] 0, T[.
$$

Then, according to (3.27) (which is still valid if we put a square on the first term), we have that $\left\|v_{n}(t)-v(t)\right\|^{2}+\left(\psi\left(v_{n}(t)\right)-\psi(v(t))\right)^{+} \rightarrow 0$ for a.e. $\left.t \in\right] 0, T$. Therefore, by dominated convergence, we get that

$$
\lim _{n \rightarrow \infty}\left(\left\|v_{n}-v\right\|_{L^{2}(0, T ; H)}^{2}+\int_{0}^{T}\left(\psi\left(v_{n}(t)\right)-\psi(v(t))\right)^{+} \mathrm{d} t\right)=0
$$

For $n \in \mathbb{N}^{\star}, k=1, \ldots, n$, for a.e. $\left.t \in\right](k-1) \tau, k \tau\left[\right.$, we let $w=v_{n}(t)$ in (3.16). We integrate on ]$(k-1) \tau, k \tau[$ and sum on $k=1, \ldots, n$. We obtain, using (3.15),

$$
\begin{aligned}
& \int_{0}^{T}\left\langle D_{t} \widehat{u}_{n}(t), v_{n}(t)-\widehat{u}_{n}(t)\right\rangle \mathrm{d} t+\int_{0}^{T}\left(\psi\left(v_{n}(t)\right)-\psi\left(\widehat{u}_{n}(t)\right)\right) \mathrm{d} t \\
\geq & \int_{0}^{T}\left\langle f_{n}(t), v_{n}(t)-\widehat{u}_{n}(t)\right\rangle \mathrm{d} t .
\end{aligned}
$$

We then notice that

$$
\begin{aligned}
& \int_{0}^{T}\left\langle D_{t} \widehat{u}_{n}(t), \widehat{u}_{n}(t)\right\rangle \mathrm{d} t=\sum_{k=1}^{n}\left\langle\widehat{u}^{k}-\widehat{u}^{k-1}, \widehat{u}^{k}\right\rangle \\
& \geq \sum_{k=1}^{n} \frac{1}{2}\left(\left\|\widehat{u}^{k}\right\|^{2}-\left\|\widehat{u}^{k-1}\right\|^{2}\right)=\frac{1}{2}\left\|\widehat{u}_{n}(T)\right\|^{2}-\frac{1}{2}\left\|\widehat{u}_{n}^{0}\right\|^{2} .
\end{aligned}
$$

According to the weak convergence of $\widehat{u}_{n}(T)$ to $u(T)$ in $H$, we have $\|u(T)\| \leq \liminf _{n \rightarrow \infty}\left\|\widehat{u}_{n}(T)\right\|$, thus using that $\widehat{u}_{n}^{0} \rightarrow u^{0}$,

$$
\limsup _{n \rightarrow \infty} \int_{0}^{T}\left\langle D_{t} \widehat{u}_{n}(t),-\widehat{u}_{n}(t)\right\rangle \mathrm{d} t \leq \frac{1}{2}\left\|u^{0}\right\|^{2}-\frac{1}{2}\|u(T)\|^{2} .
$$

But using the weak convergence of $D_{t} \widehat{u}_{n}$ to $\partial_{t} u$ in $L^{2}(0, T ; H)$ and (3.33), we have

$$
\lim _{n \rightarrow \infty} \int_{0}^{T}\left\langle D_{t} \widehat{u}_{n}(t), v_{n}(t)\right\rangle \mathrm{d} t=\int_{0}^{T}\left\langle\partial_{t} u(t), v(t)\right\rangle \mathrm{d} t .
$$


According to Lemma 5.5 given in the appendix, (3.33) implies that $\psi\left(v_{n}(t)\right) \rightarrow \psi(v(t))$ in $L^{1}(0, T)$. Thus using Fatou's lemma and the lower semi-continuity of $\psi$,

$$
\limsup _{n \rightarrow \infty} \int_{0}^{T}\left(\psi\left(v_{n}(t)\right)-\psi\left(\widehat{u}_{n}(t)\right)\right) \mathrm{d} t \leq \int_{0}^{T}(\psi(v(t))-\psi(u(t))) \mathrm{d} t .
$$

Using the density of $C^{1}([0, T] ; H)$ in $L^{2}(0, T ; H)$, we get the convergence of $f_{n}$ to $f$ strongly in $L^{2}(0, T ; H)$. Therefore we can then pass to the limit in $(3.34)$, and get

$$
\begin{aligned}
& \int_{0}^{T}\left\langle\partial_{t} u(t), v(t)\right\rangle \mathrm{d} t+\frac{1}{2}\left\|u^{0}\right\|^{2}-\frac{1}{2}\|u(T)\|^{2}+\int_{0}^{T}(\psi(v(t))-\psi(u(t))) \mathrm{d} t \\
\geq & \int_{0}^{T}\langle f(t), v(t)-u(t)\rangle \mathrm{d} t .
\end{aligned}
$$

Since

$$
\int_{0}^{T}\left\langle\partial_{t} u(t), u(t)\right\rangle \mathrm{d} t=\frac{1}{2}\|u(T)\|^{2}-\frac{1}{2}\left\|u^{0}\right\|^{2}
$$

we obtain that $u$ satisfies (3.3). According to the uniqueness of the solution to (3.3), we conclude that the whole sequence converges.

The next lemma states an error estimate for this approximate method.

Lemma 3.9 Under Hypothesis 2.6, let $T>0, f \in L^{2}(0, T ; H)$ and $u^{0} \in B$ be given, and let $u$ denote the unique solution of Problem (3.3). Let $\widehat{H}$ be a closed subspace of $H$, and let $\widehat{B}=\widehat{H} \cap B$. For a given $n \in \mathbb{N}^{\star}$, let $\tau=T / n$, let $\widehat{u}$ and $D_{t} \widehat{u}$ be defined by (3.14)-(3.18). Then, there exists an absolute constant $C_{0} \geq 0$ such that

$$
\|\widehat{u}(t)-u(t)\|^{2} \leq C_{0}\left(C_{2} \tau+T\left\|f_{n}-f\right\|_{L^{2}(0, T ; H)}^{2}+\left\|\widehat{u}^{0}-u^{0}\right\|^{2}+\widehat{\mathcal{R}}_{u}\right), \forall t \in[0, T],
$$

and

$$
\begin{aligned}
& \int_{0}^{T}|\psi(\widehat{u}(t))-\psi(u(t))| \mathrm{d} t \\
\leq & \widehat{\mathcal{R}}_{u}+C_{0}\left(T C_{2}\right)^{1 / 2}\left(C_{2} \tau+T\left\|f_{n}-f\right\|_{L^{2}(0, T ; H)}^{2}+\left\|\widehat{u}^{0}-u^{0}\right\|^{2}+\widehat{\mathcal{R}}_{u}\right)^{1 / 2},
\end{aligned}
$$

where $f_{n}(t)$ is defined by (3.15), $C_{2}$ is defined by (3.25), and

$$
\begin{gathered}
\widehat{\mathcal{R}}_{u}=\inf _{v \in L^{2}(0, T ; \widehat{H}) \cap B_{T}}\left(\left((2 G)^{1 / 2}+2\|f\|_{L^{2}(0, T ; H)}\right)\|v-u\|_{L^{2}(0, T ; H)}\right. \\
\left.+\int_{0}^{T}(\psi(v(t))-\psi(u(t)))^{+} \mathrm{d} t\right) .
\end{gathered}
$$

Remark 3.10 The estimate (3.35) extends [14, Theorem 3] to the case of time dependent righthand side. We use in (3.35) the continuity properties of the functions with respect to $t$.

Proof of Lemma 3.9. The assumptions enable to apply Lemma 3.5, thus we have the estimates (3.19)-(3.22). As in the proof of Lemma 2.8, we consider, for a given $v \in L^{2}(0, T ; \widehat{H}) \cap$ $B_{T}$, the test function $w=v(t)$ in (3.16), for a.e. $\left.t \in\right](k-1) \tau, k \tau[$. This gives that

$$
\left.\left\langle D_{t} \widehat{u}(t), v(t)-\widehat{u}(t)\right\rangle+\psi(v(t))-\psi(\widehat{u}(t)) \geq\left\langle f_{n}(t), v(t)-\widehat{u}(t)\right\rangle \text {, for a.e. } t \in\right] 0, T[.
$$


For a given time $t_{2} \in[0, T]$, we integrate the previous inequality on $] 0, t_{2}[$, and obtain

$$
\begin{aligned}
& \int_{0}^{t_{2}}\left\langle D_{t} \widehat{u}(t), u(t)-\widehat{u}(t)\right\rangle \mathrm{d} t+\int_{0}^{t_{2}}(\psi(u(t))-\psi(\widehat{u}(t))) \mathrm{d} t+R_{n} \\
& \geq \int_{0}^{t_{2}}\left\langle f_{n}(t), u(t)-\widehat{u}(t)\right\rangle \mathrm{d} t,
\end{aligned}
$$

with

$$
R_{n}=\int_{0}^{t_{2}}\left\langle D_{t} \widehat{u}(t)-f_{n}(t), v(t)-u(t)\right\rangle \mathrm{d} t+\int_{0}^{t_{2}}(\psi(v(t))-\psi(u(t))) \mathrm{d} t .
$$

We then get, according to (3.23) and (3.20),

$$
R_{n} \leq\left(\left(C_{2}\right)^{1 / 2}+\|f\|_{L^{2}(0, T ; H)}\right)\|v-u\|_{L^{2}(0, T ; H)}+\int_{0}^{T}(\psi(v(t))-\psi(u(t)))^{+} \mathrm{d} t .
$$

Taking $\widehat{u}$ as test function in (3.4) with $t_{1}=0$, we get

$$
\begin{aligned}
& \int_{0}^{t_{2}}\left\langle\partial_{t} u(t), \widehat{u}(t)-u(t)\right\rangle \mathrm{d} t+\int_{0}^{t_{2}}(\psi(\widehat{u}(t))-\psi(u(t))) \mathrm{d} t \\
\geq & \int_{0}^{t_{2}}\langle f(t), \widehat{u}(t)-u(t)\rangle \mathrm{d} t .
\end{aligned}
$$

The sum of (3.39) and (3.41) gives

$$
\int_{0}^{t_{2}}\left\langle D_{t} \widehat{u}(t)-\partial_{t} u(t), u(t)-\widehat{u}(t)\right\rangle \mathrm{d} t+R_{n} \geq \int_{0}^{t_{2}}\left\langle f_{n}(t)-f(t), u(t)-\widehat{u}(t)\right\rangle \mathrm{d} t .
$$

We now introduce the function $\widetilde{u}(t)$ defined by

$$
\widetilde{u}(t)=\frac{t-(k-1) \tau}{\tau} \widehat{u}^{k}+\frac{k \tau-t}{\tau} \widehat{u}^{k-1}, \forall t \in[(k-1) \tau, k \tau], \forall k=1, \ldots, n,
$$

so that

$$
\left.\partial_{t} \widetilde{u}(t)=D_{t} \widehat{u}(t) \text {, for a.e. } t \in\right] 0, T[\text {, }
$$

which yields

$$
\int_{0}^{t_{2}}\left\langle D_{t} \widehat{u}(t)-\partial_{t} u(t), u(t)-\widetilde{u}(t)\right\rangle \mathrm{d} t=\frac{1}{2}\left\|\widehat{u}^{0}-u^{0}\right\|^{2}-\frac{1}{2}\left\|\widetilde{u}\left(t_{2}\right)-u\left(t_{2}\right)\right\|^{2} .
$$

This leads to

$$
\begin{aligned}
& \frac{1}{2}\left\|\widehat{u}^{0}-u^{0}\right\|^{2}-\frac{1}{2}\left\|\widetilde{u}\left(t_{2}\right)-u\left(t_{2}\right)\right\|^{2} \\
& +\int_{0}^{t_{2}}\left\langle D_{t} \widehat{u}(t)-\partial_{t} u(t), \widetilde{u}(t)-\widehat{u}(t)\right\rangle \mathrm{d} t+R_{n} \\
\geq & \int_{0}^{t_{2}}\left\langle f_{n}(t)-f(t), u(t)-\widehat{u}(t)\right\rangle \mathrm{d} t .
\end{aligned}
$$

We have for $t \in](k-1) \tau, k \tau]$

$$
\widetilde{u}(t)-\widehat{u}(t)=\frac{t-k \tau}{\tau}\left(\widehat{u}^{k}-\widehat{u}^{k-1}\right)=\frac{t-k \tau}{\tau}(\widehat{u}(k \tau)-\widehat{u}((k-1) \tau)),
$$


and thus using (3.22),

$$
\|\widetilde{u}(t)-\widehat{u}(t)\| \leq\left(2 C_{2} \tau\right)^{1 / 2}, \forall t \in[0, T] .
$$

We also have

$$
\|\widetilde{u}(t)-\widehat{u}(t)\| \leq \tau\left\|D_{t} \widehat{u}(t)\right\|, \forall t \in[0, T] .
$$

Using the estimates (3.20), (3.30), we thus get

$$
\begin{aligned}
\frac{1}{4}\left\|\widehat{u}\left(t_{2}\right)-u\left(t_{2}\right)\right\|^{2} \leq & \frac{1}{2}\left\|\widehat{u}\left(t_{2}\right)-\widetilde{u}\left(t_{2}\right)\right\|^{2}+\frac{1}{2}\left\|\widetilde{u}\left(t_{2}\right)-u\left(t_{2}\right)\right\|^{2} \\
\leq & C_{2} \tau+\frac{1}{2}\left\|\widehat{u}^{0}-u^{0}\right\|^{2}+2\left(C_{2}\right)^{1 / 2} \tau\left(C_{2}\right)^{1 / 2}+R_{n} \\
& +\left\|f_{n}-f\right\|_{L^{2}(0, T ; H)}\left(\int_{0}^{t_{2}}\|\widehat{u}(t)-u(t)\|^{2} \mathrm{~d} t\right)^{1 / 2} .
\end{aligned}
$$

Since this holds for all $t_{2} \in[0, T]$, with the Gronwall argument of Lemma 3.2 we deduce that for all $t \in[0, T]$

$$
\frac{1}{4}\|\widehat{u}(t)-u(t)\|^{2} \leq 2\left(3 C_{2} \tau+\frac{1}{2}\left\|\widehat{u}^{0}-u^{0}\right\|^{2}+R_{n}\right)+8 T\left\|f_{n}-f\right\|_{L^{2}(0, T ; H)}^{2},
$$

which shows (3.35) with (3.40), since $v \in L^{2}(0, T ; \widehat{H}) \cap B_{T}$ is arbitrary. We next deduce from (3.38) that for all $v \in L^{2}(0, T ; \widehat{H}) \cap B_{T}$ and a.e. $\left.t \in\right] 0, T[$,

$$
\begin{aligned}
& \psi(\widehat{u}(t))-\psi(v(t)) \leq\left\langle D_{t} \widehat{u}(t)-f_{n}(t), u(t)-\widehat{u}(t)\right\rangle \\
&+\left\|D_{t} \widehat{u}(t)-f_{n}(t)\right\|\|v(t)-u(t)\| .
\end{aligned}
$$

We symmetrically deduce from (3.5) in which we take $\widehat{u}$ as test function, that for a.e. $t \in] 0, T[$

$$
\psi(u(t))-\psi(\widehat{u}(t)) \leq\left\langle\partial_{t} u(t)-f(t), \widehat{u}(t)-u(t)\right\rangle .
$$

This leads to

$$
\begin{aligned}
& |\psi(\widehat{u}(t))-\psi(u(t))| \\
\leq & \left(\left\|\partial_{t} u(t)\right\|+\left\|D_{t} \widehat{u}(t)\right\|+\left\|f_{n}(t)\right\|+\|f(t)\|\right)\|\widehat{u}(t)-u(t)\| \\
& +\left\|D_{t} \widehat{u}(t)-f_{n}(t)\right\|\|v(t)-u(t)\|+(\psi(v(t))-\psi(u(t)))^{+} .
\end{aligned}
$$

Integrating this relation on $] 0, T[$, using (3.35), the Cauchy-Schwarz inequality and taking the infimum on $v \in L^{2}(0, T ; \widehat{H}) \cap B_{T}$ provides (3.36).

With Lemma 3.9, we now deduce the following convergence result for the approximate method.

Theorem 3.11 Under the hypotheses of Theorem 3.6, $\widehat{u}_{n}(t)$ converges strongly in $H$ to $u(t) \in B$ as $n$ tends to $\infty$, uniformly in $t \in[0, T]$, and $\psi\left(\widehat{u}_{n}\right)$ converges in $L^{1}(] 0, T[)$ to $\psi(u)$.

Proof. We can apply Lemma 3.9 with $G=\sup _{n} \psi\left(\widehat{u}_{n}^{0}\right)$. Let us prove that the right-hand sides of (3.35) and (3.36) tend to 0 as $n \rightarrow \infty$. It is clear that $\tau \rightarrow 0$ as $n \rightarrow \infty$. We recall that by density of $C^{1}([0, T] ; H)$ in $L^{2}(0, T ; H)$, we have the strong convergence of $f_{n}$ to $f$ in $L^{2}(0, T ; H)$. Therefore it only remains to prove that

$$
\inf _{v \in L^{2}\left(0, T ; \widehat{H}_{n}\right) \cap B_{T}}\left(\|v-u\|_{L^{2}(0, T ; H)}+\int_{0}^{T}(\psi(v(t))-\psi(u(t)))^{+} \mathrm{d} t\right) \longrightarrow 0,
$$


as $n \rightarrow \infty$. Let us prove this property for all $u \in C^{0}([0, T] ; H)$ such that $u(t) \in B$ for all $t \in[0, T]$. Given $\eta>0$, consider an integer $M \in \mathbb{N}^{\star}$, and for $i=1, \ldots, M$ the intervals $I_{i}=[(i-1) T / M, i T / M]$. Then $K_{i}=u\left(I_{i}\right)$ is a compact subset of H. Since $\psi$ is lower semicontinuous, it attains its lower bound over each $K_{i}$. In other words, there exists $t_{i} \in I_{i}$ such that

$$
\psi(u(t)) \geq \psi\left(u\left(t_{i}\right)\right), \quad \forall t \in I_{i} .
$$

Since $u$ is uniformly continuous, one can choose $M \in \mathbb{N}^{\star}$ such that

$$
\left\|u\left(t_{i}\right)-u(t)\right\| \leq \eta, \quad \forall t \in I_{i}, \forall i=1, \ldots, M .
$$

Then applying the property $(3.27)$ to $u\left(t_{i}\right)$ for all $i=1, \ldots, M$, we find $n_{1}$ such that for all $n \geq n_{1}$, we can find $w_{i} \in \widehat{B}_{n}$ for $i=1, \ldots, M$ satisfying

$$
\left\|w_{i}-u\left(t_{i}\right)\right\|+\left(\psi\left(w_{i}\right)-\psi\left(u\left(t_{i}\right)\right)\right)^{+} \leq \eta .
$$

Define then the function $v$ by

$$
\left.\left.v(t)=w_{i}, \quad \forall t \in\right](i-1) T / M, i T / M\right], \forall i=1, \ldots, M,
$$

and $v(0)=w_{1}$. Then $v \in L^{2}\left(0, T ; \widehat{H}_{n}\right) \cap B_{T}$,

$$
\sup _{t \in[0, T]}\|v(t)-u(t)\| \leq 2 \eta, \quad \sup _{t \in[0, T]}(\psi(v(t))-\psi(u(t)))^{+} \leq \eta,
$$

which concludes the proof of (3.43).

\subsection{Total variation functionals, regularized implicit approximations}

We now take a functional $\psi$ of the type considered in Subsection 2.2, as stated in Hypothesis 2.10 , and we modify the scheme (3.14)-(3.18) by including a regularization procedure with parameter $\varepsilon>0$. We assume that $\widehat{H}$ is a finite dimensional subspace of $H_{1}$, that $\widehat{u}^{0} \in \widehat{H}$ is an approximation of $u^{0} \in B$. For a given $n \in \mathbb{N}^{\star}$, we define $\tau=T / n$ and the sequence $\left(\widehat{u}_{\varepsilon}^{k}\right)_{k=1, \ldots, n}$ by

$$
\begin{aligned}
& \widehat{u}_{\varepsilon}^{k} \in \widehat{H}, \\
& \left\langle D^{k} \widehat{u}_{\varepsilon}, w\right\rangle+\int_{\Omega} \frac{A\left(\widehat{u}_{\varepsilon}^{k}, w\right)(x)}{\varepsilon+a\left(\widehat{u}_{\varepsilon}^{k}\right)(x)} \mathrm{d} \mu=\left\langle f^{k}, w\right\rangle, \forall w \in \widehat{H}, \forall k=1, \ldots, n,
\end{aligned}
$$

again using (3.17) (with index $\varepsilon$ ) and (3.15). This scheme is called the regularized implicit algorithm. At each timestep, a problem of the form (2.31) with $\alpha=1 / \tau, f=f^{k}+\widehat{u}_{\varepsilon}^{k-1} / \tau$ has to be solved, and the fixed point method (2.43) can be used. Note that, according to Lemma 2.13 , there exists one and only one family $\left(\widehat{u}_{\varepsilon}^{k}\right)_{k=1, \ldots, n}$ defined by $(3.46)$.

Theorem 3.12 Under Hypothesis 2.10, let $T>0, f \in L^{2}(0, T ; H)$ and $u^{0} \in B$ be given. Let $\widehat{H}$ be a finite dimensional subspace of $H_{1}$, and let $\widehat{B}=\widehat{H}$. Let $\varepsilon>0$ be given, and, for $n \in \mathbb{N}^{\star}$, let $\tau=T / n$, let $\widehat{u}_{\varepsilon}$ be defined by (3.14), (3.15), (3.46), and (3.17), (3.18) with indices $\varepsilon$ everywhere, and let $\widehat{u}$ be defined by (3.14)-(3.18), with $\widehat{u}_{\varepsilon}^{0}=\widehat{u}^{0}$. Then it holds

$$
\left\|\widehat{u}_{\varepsilon}(t)-\widehat{u}(t)\right\|^{2} \leq 2 \varepsilon \mu(\Omega) T, \forall t \in[0, T],
$$


and

$$
\int_{0}^{T}\left|\psi\left(\widehat{u}_{\varepsilon}(t)\right)-\psi(\widehat{u}(t))\right| \mathrm{d} t \leq \varepsilon \mu(\Omega) T+\left(2 G^{1 / 2}+3\|f\|_{L^{2}(0, T ; H)}\right)\left(2 \varepsilon \mu(\Omega) T^{2}\right)^{1 / 2} .
$$

Remark 3.13 By Lemma 3.9 and the triangle inequality, we get estimates on $\left\|\widehat{u}_{\varepsilon}(t)-u(t)\right\|$ and on $\int\left|\psi\left(\widehat{u}_{\varepsilon}(t)\right)-\psi(u(t))\right| d t$. These are improvements with respect to [13, Theorem 1.7] since Theorem 3.12 does not include terms in $1 / \varepsilon$ (thus the limit $\varepsilon \rightarrow 0$ is possible), and no additional regularity is required on the solution. Moreover, under assumptions (3.27), (3.28), Theorem 3.11 gives also the convergence of $\widehat{u}_{\varepsilon}$ to $u$ as $\varepsilon \rightarrow 0$ and $n \rightarrow \infty$.

Proof of Theorem 3.12. Using (5.6) (in the same way as in (2.39)), we get from (3.46)

$$
\left\langle f^{k}, v-\widehat{u}_{\varepsilon}^{k}\right\rangle \leq\left\langle D^{k} \widehat{u}_{\varepsilon}, v-\widehat{u}_{\varepsilon}^{k}\right\rangle+\psi(v)-\psi\left(\widehat{u}_{\varepsilon}^{k}\right)+\varepsilon \mu(\Omega), \forall v \in \widehat{H} .
$$

We then let $v=\widehat{u}^{k}$, and we add the result to (3.16), with $w=\widehat{u}_{\varepsilon}^{k}$. We thus obtain

$$
\left\langle D^{k}\left(\widehat{u}_{\varepsilon}-\widehat{u}\right), \widehat{u}_{\varepsilon}^{k}-\widehat{u}^{k}\right\rangle \leq \varepsilon \mu(\Omega) .
$$

Multiplying by $\tau$, using $(a-b) a=\frac{1}{2}\left(a^{2}+(a-b)^{2}-b^{2}\right)$ and summing on $k=1, \ldots, m$ for $m=1, \ldots, n$, we get

$$
\frac{1}{2}\left\|\widehat{u}_{\varepsilon}^{m}-\widehat{u}^{m}\right\|^{2} \leq \frac{1}{2}\left\|\widehat{u}_{\varepsilon}^{0}-\widehat{u}^{0}\right\|^{2}+\varepsilon \mu(\Omega) T,
$$

which proves (3.47).

We then have, taking $w=\widehat{u}_{\varepsilon}^{k}-\widehat{u}_{\varepsilon}^{k-1}$ in (3.46), for $k=1, \ldots, n$, and omitting $(x)$,

$$
\tau\left\|D^{k} \widehat{u}_{\varepsilon}\right\|^{2}+\int_{\Omega} \frac{A\left(\widehat{u}_{\varepsilon}^{k}, \widehat{u}_{\varepsilon}^{k}-\widehat{u}_{\varepsilon}^{k-1}\right)}{\varepsilon+a\left(\widehat{u}_{\varepsilon}^{k}\right)} \mathrm{d} \mu=\tau\left\langle f^{k}, D^{k} \widehat{u}_{\varepsilon}\right\rangle .
$$

We now use the Cauchy-Schwarz inequality, which implies

$$
a\left(\widehat{u}_{\varepsilon}^{k}\right)\left(a\left(\widehat{u}_{\varepsilon}^{k}\right)-a\left(\widehat{u}_{\varepsilon}^{k-1}\right)\right) \leq A\left(\widehat{u}_{\varepsilon}^{k}, \widehat{u}_{\varepsilon}^{k}-\widehat{u}_{\varepsilon}^{k-1}\right),
$$

and we use (5.5). This leads to

$$
\tau\left\|D^{k} \widehat{u}_{\varepsilon}\right\|^{2}+\int_{\Omega}\left(F_{\varepsilon}\left(a\left(\widehat{u}_{\varepsilon}^{k}\right)\right)-F_{\varepsilon}\left(a\left(\widehat{u}_{\varepsilon}^{k-1}\right)\right)\right) \mathrm{d} \mu \leq \tau\left\langle f^{k}, D^{k} \widehat{u}_{\varepsilon}\right\rangle \leq \frac{\tau}{2}\left\|f^{k}\right\|^{2}+\frac{\tau}{2}\left\|D^{k} \widehat{u}_{\varepsilon}\right\|^{2} .
$$

Hence, taking the sum on $k=1, \ldots, n$, using $\int_{\Omega} F_{\varepsilon}\left(a\left(\widehat{u}_{\varepsilon}^{0}\right)\right) \mathrm{d} \mu \leq \int_{\Omega} a\left(\widehat{u}_{\varepsilon}^{0}\right) \mathrm{d} \mu=\psi\left(\widehat{u}_{\varepsilon}^{0}\right) \leq G$, we get that, for $C_{2}=2 G+\|f\|_{L^{2}(0, T ; H)}^{2}$, one has

$$
\left\|D_{t} \widehat{u}_{\varepsilon}\right\|_{L^{2}(0, T ; H)}^{2} \leq C_{2} .
$$

We get, as above from (3.49) with $v=\widehat{u}^{k}$, and (3.16) with $w=\widehat{u}_{\varepsilon}^{k}$, that

$$
\left|\psi\left(\widehat{u}_{\varepsilon}^{k}\right)-\psi\left(\widehat{u}^{k}\right)\right| \leq \varepsilon \mu(\Omega)+\left|\left\langle f^{k}, \widehat{u}_{\varepsilon}^{k}-\widehat{u}^{k}\right\rangle\right|+\max \left(\left|\left\langle D^{k} \widehat{u}_{\varepsilon}, \widehat{u}_{\varepsilon}^{k}-\widehat{u}^{k}\right\rangle\right|,\left|\left\langle D^{k} \widehat{u}, \widehat{u}_{\varepsilon}^{k}-\widehat{u}^{k}\right\rangle\right|\right) .
$$

Writing the corresponding inequality for all $t \in[0, T]$ and integrating the result on $[0, T]$, using the Cauchy-Schwarz inequality, (3.47), (3.20) and (3.50), we get (3.48). 


\subsection{Total variation functionals, regularized linearized implicit approxima- tions}

As in Subsection 3.3, we again take a functional $\psi$ of the type considered in Subsection 2.2, as stated in Hypothesis 2.10, we again modify the scheme (3.14)-(3.18) by including a regularization procedure with parameter $\varepsilon>0$. We assume that $\widehat{H}$ is a finite dimensional subspace of $H_{1}$, that $\widehat{u}^{0} \in \widehat{H}$ is an approximation of $u^{0} \in B$. For a given $n \in \mathbb{N}^{\star}$, we define $\tau=T / n$ and the sequence $\left(\widehat{u}^{k}\right)_{k=1, \ldots, n}$ by

$$
\begin{aligned}
& \widehat{u}^{k} \in \widehat{H}, \\
& \left\langle D^{k} \widehat{u}, w\right\rangle+\int_{\Omega} \frac{A\left(\widehat{u}^{k}, w\right)(x)}{\varepsilon+a\left(\widehat{u}^{k-1}\right)(x)} \mathrm{d} \mu=\left\langle f^{k}, w\right\rangle, \forall w \in \widehat{H}, \forall k=1, \ldots, n,
\end{aligned}
$$

using (3.17) and (3.15). Note that the main difference with Subsection 3.3 is that Problem (3.51) has been linearized with respect to $\widehat{u}^{k}$, the denominator being evaluated at $\widehat{u}^{k-1}$, corresponding to the previous time step. This is what we call the regularized linearized implicit algorithm. The great advantage of the approximation (3.51) with respect to (3.46) is that at each time step it suffices to solve a linear system. Then the limitation that $\varepsilon$ must not be too small in Lemma 2.15 (otherwise the convergence of the iterative method is very slow) is replaced here by a restriction saying that the time step must be much smaller than $\varepsilon$. Moreover, the assumption (3.27) (or equivalently (2.34), (2.35)) has to be slightly strengthened.

Theorem 3.14 Under Hypothesis 2.10, let $T>0, f \in L^{2}(0, T ; H)$ and $u^{0} \in B$ be given, and let $u$ denote the unique solution of Problem (3.3). Let $\left(\widehat{H}_{n}\right)_{n \in \mathbb{N}^{\star}}$ be a sequence of finite dimensional subspaces of $H_{1}$. We assume that

$$
\inf _{w \in H_{1}}\left(\|w-v\|+(\psi(w)-\psi(v))^{+}\right)=0, \forall v \in B
$$

and that

$$
\lim _{n \rightarrow \infty} \inf _{w \in \widehat{H}_{n}}\left(\|w-v\|+\left(\int_{\Omega}(a(w-v)(x))^{2} \mathrm{~d} \mu(x)\right)^{1 / 2}\right)=0, \forall v \in H_{1} .
$$

For all $n \in \mathbb{N}^{\star}$, let $\tau_{n}=T / n$, let $\widehat{u}_{n}^{0} \in \widehat{H}_{n}$ be such that

$$
\left\|\widehat{u}_{n}^{0}-u^{0}\right\| \rightarrow 0, \text { as } n \rightarrow \infty, \quad \sup _{n} \psi\left(\widehat{u}_{n}^{0}\right)<\infty .
$$

Let $\left(\varepsilon_{n}\right)_{n \in \mathbb{N}^{\star}}$ be a sequence of positive numbers converging to 0 , such that

$$
\frac{\tau_{n}}{\varepsilon_{n}} \longrightarrow 0, \text { as } n \rightarrow \infty
$$

Let $\widehat{u}_{n}$ and $D_{t} \widehat{u}_{n}$ be defined by $\widehat{u}^{0}=\widehat{u}_{n}^{0}$, (3.17), (3.18), (3.15), and (3.51) where $\widehat{H}$ has to be replaced by $\widehat{H}_{n}, \tau$ by $\tau_{n}$, and $\varepsilon$ by $\varepsilon_{n}$. Then $\widehat{u}_{n}(t)$ converges in $H$ to $u(t) \in B$ as $n$ tends to $\infty$, uniformly in $t \in[0, T]$, and $\psi\left(\widehat{u}_{n}\right)$ converges in $L^{1}(] 0, T[)$ to $\psi(u)$.

Proof. Let us first notice that (3.52) and (3.53) imply (2.34) and (2.35), because $|\psi(w)-\psi(v)|=$ $\left|\int_{\Omega}(a(w)-a(v)) d \mu\right| \leq \int_{\Omega} a(w-v) d \mu \leq\left(\mu(\Omega) \int_{\Omega} a(w-v)^{2} d \mu\right)^{1 / 2}$, and thus also imply (3.27). 
We denote by $G \geq 0$ a constant such that $\psi\left(\widehat{u}_{n}^{0}\right) \leq G$. We have, taking $w=\widehat{u}^{k}-\widehat{u}^{k-1}$ in (3.51), for $k=1, \ldots, n$,

$$
\tau_{n}\left\|D^{k} \widehat{u}\right\|^{2}+\int_{\Omega} \frac{A\left(\widehat{u}^{k}, \widehat{u}^{k}-\widehat{u}^{k-1}\right)(x)}{\varepsilon_{n}+a\left(\widehat{u}^{k-1}\right)(x)} \mathrm{d} \mu=\tau_{n}\left\langle f^{k}, D^{k} \widehat{u}\right\rangle .
$$

We again use (5.4) proved in Lemma 5.1. This leads, omitting $(x)$, to

$$
\begin{aligned}
& \tau_{n}\left\|D^{k} \widehat{u}\right\|^{2}+\int_{\Omega}\left(F_{\varepsilon_{n}}\left(a\left(\widehat{u}^{k}\right)\right)-F_{\varepsilon_{n}}\left(a\left(\widehat{u}^{k-1}\right)\right)+\frac{\left(a\left(\widehat{u}^{k}\right)-a\left(\widehat{u}^{k-1}\right)\right)^{2}}{2\left(\varepsilon_{n}+a\left(\widehat{u}^{k-1}\right)\right)}\right) \mathrm{d} \mu \\
& \leq \tau_{n}\left\langle f^{k}, D^{k} \widehat{u}\right\rangle \leq \frac{\tau_{n}}{2}\left\|f^{k}\right\|^{2}+\frac{\tau_{n}}{2}\left\|D^{k} \widehat{u}\right\|^{2} .
\end{aligned}
$$

Hence, taking the sum on $k=1, \ldots, n$, using (5.3) and $\int_{\Omega} F_{\varepsilon_{n}}\left(a\left(\widehat{u}^{0}\right)\right) \mathrm{d} \mu \leq \int_{\Omega} a\left(\widehat{u}_{n}^{0}\right) \mathrm{d} \mu=\psi\left(\widehat{u}_{n}^{0}\right) \leq$ $G$, we get the existence of $C_{2} \geq 0$, only depending on $\|f\|_{L^{2}(0, T ; H)}$ and $G$, such that

$$
\begin{gathered}
\sum_{k=1}^{n} \int_{\Omega} \frac{\left(a\left(\widehat{u}^{k}\right)-a\left(\widehat{u}^{k-1}\right)\right)^{2}}{2\left(\varepsilon_{n}+a\left(\widehat{u}^{k-1}\right)\right)} \mathrm{d} \mu \leq C_{2}, \\
\left\|D_{t} \widehat{u}_{n}\right\|_{L^{2}(0, T ; H)}^{2} \leq C_{2}, \\
\psi\left(\widehat{u}_{n}(t)\right) \leq C_{2}+\varepsilon_{n} \mu(\Omega), \forall t \in[0, T], \\
\left\|\widehat{u}_{n}(t)\right\| \leq\left\|\widehat{u}_{n}^{0}\right\|+\left(T C_{2}\right)^{1 / 2}, \forall t \in[0, T],
\end{gathered}
$$

and

$$
\left\|\widehat{u}_{n}\left(t_{2}\right)-\widehat{u}_{n}\left(t_{1}\right)\right\| \leq\left(C_{2}\right)^{1 / 2}\left(\left|t_{2}-t_{1}\right|+\tau_{n}\right)^{1 / 2}, \forall t_{1}, t_{2} \in[0, T] .
$$

Another a priori estimate is needed in further computations. We let $w=\tau_{n} \widehat{u}^{k}$ in (3.51), for $k=1, \ldots, n$, and we take the sum on $k=1, \ldots, n$. We obtain, using the inequality $\frac{1}{2} a^{2}-\frac{1}{2} b^{2} \leq$ $(a-b) a$,

$$
\frac{1}{2}\left\|\widehat{u}^{n}\right\|^{2}-\frac{1}{2}\left\|\widehat{u}_{n}^{0}\right\|^{2}+\sum_{k=1}^{n} \tau_{n} \int_{\Omega} \frac{a\left(\widehat{u}^{k}\right)^{2}}{\varepsilon_{n}+a\left(\widehat{u}^{k-1}\right)} \mathrm{d} \mu \leq \sum_{k=1}^{n} \tau_{n}\left\langle f^{k}, \widehat{u}^{k}\right\rangle .
$$

We then apply (3.59) and (3.23). We obtain

$$
\sum_{k=1}^{n} \tau_{n} \int_{\Omega} \frac{a\left(\widehat{u}^{k}\right)^{2}}{\varepsilon_{n}+a\left(\widehat{u}^{k-1}\right)} \mathrm{d} \mu \leq \frac{1}{2}\left\|\widehat{u}_{n}^{0}\right\|^{2}+\|f\|_{L^{2}(0, T ; H)} T^{1 / 2}\left(\left\|\widehat{u}_{n}^{0}\right\|+\left(T C_{2}\right)^{1 / 2}\right) \leq C_{1},
$$

for some constant $C_{1}$. For a given element $v_{n} \in L^{2}\left(0, T ; \widehat{H}_{n}\right) \cap B_{T}$, we let $w=v_{n}(t)-\widehat{u}^{k}$ in (3.51), for a.e. $t \in](k-1) \tau_{n}, k \tau_{n}[$ and $k=1, \ldots, n$. This gives that

$$
\left\langle D^{k} \widehat{u}, v_{n}(t)-\widehat{u}^{k}\right\rangle+\int_{\Omega} \frac{A\left(\widehat{u}^{k}, v_{n}(t)-\widehat{u}^{k}\right)}{\varepsilon_{n}+a\left(\widehat{u}^{k-1}\right)} \mathrm{d} \mu=\left\langle f^{k}, v_{n}(t)-\widehat{u}^{k}\right\rangle,
$$

which provides, according to the Cauchy-Schwarz inequality,

$$
\left\langle D^{k} \widehat{u}, v_{n}(t)-\widehat{u}^{k}\right\rangle+\int_{\Omega} \frac{a\left(\widehat{u}^{k}\right)\left(a\left(v_{n}(t)\right)-a\left(\widehat{u}^{k}\right)\right)}{\varepsilon_{n}+a\left(\widehat{u}^{k-1}\right)} \mathrm{d} \mu \geq\left\langle f^{k}, v_{n}(t)-\widehat{u}^{k}\right\rangle .
$$

Then, for a given $\left.\left.t_{2} \in\right] 0, T\right]$, we take $m=1, \ldots, n$ so that $(m-1) \tau_{n}<t_{2} \leq m \tau_{n}$, we integrate the previous equation on $](k-1) \tau_{n}, k \tau_{n}[$, sum on $k=1, \ldots, m-1$, and also sum the integral 
over $](m-1) \tau_{n}, t_{2}\left[\right.$ for $k=m$. This leads, using (5.7) with $c=a\left(\widehat{u}^{k-1}\right)(x), d=a\left(\widehat{u}^{k}\right)(x)$ and $e=a\left(v_{n}(t)\right)(x)$, to

$$
\begin{aligned}
& \int_{0}^{t_{2}}\left\langle f_{n}(t), v_{n}(t)-\widehat{u}_{n}(t)\right\rangle \mathrm{d} t \\
& \leq \int_{0}^{t_{2}}\left(\left\langle D_{t} \widehat{u}_{n}(t), v_{n}(t)-\widehat{u}_{n}(t)\right\rangle+\psi\left(v_{n}(t)\right)-\psi\left(\widehat{u}_{n}(t)\right)\right) \mathrm{d} t \\
& \quad+\varepsilon_{n} \mu(\Omega) T+T_{n}^{(1)}+T_{n}^{(2)},
\end{aligned}
$$

with

$$
T_{n}^{(1)}=\sum_{k=1}^{n} \int_{(k-1) \tau_{n}}^{k \tau_{n}} \int_{\Omega} \frac{a\left(v_{n}(t)\right)\left|a\left(\widehat{u}^{k}\right)-a\left(\widehat{u}^{k-1}\right)\right|}{\varepsilon_{n}+a\left(\widehat{u}^{k-1}\right)} \mathrm{d} \mu \mathrm{d} t
$$

and

$$
T_{n}^{(2)}=\tau_{n} \sum_{k=1}^{n} \int_{\Omega} \frac{a\left(\widehat{u}^{k}\right)\left|a\left(\widehat{u}^{k}\right)-a\left(\widehat{u}^{k-1}\right)\right|}{\varepsilon_{n}+a\left(\widehat{u}^{k-1}\right)} \mathrm{d} \mu .
$$

We have, according to the Cauchy-Schwarz inequality and to (3.56),

$$
\begin{aligned}
\left(T_{n}^{(1)}\right)^{2} & \leq \frac{1}{\varepsilon_{n}}\left\|a\left(v_{n}\right)\right\|_{L^{2}\left(0, T ; L_{\mu}^{2}(\Omega)\right)}^{2} \sum_{k=1}^{n} \tau_{n} \int_{\Omega} \frac{\left(a\left(\widehat{u}^{k}\right)-a\left(\widehat{u}^{k-1}\right)\right)^{2}}{\varepsilon_{n}+a\left(\widehat{u}^{k-1}\right)} \mathrm{d} \mu \\
& \leq 2 C_{2} \frac{\tau_{n}}{\varepsilon_{n}}\left\|a\left(v_{n}\right)\right\|_{L^{2}\left(0, T ; L_{\mu}^{2}(\Omega)\right)}^{2} .
\end{aligned}
$$

We then have similarly, using (3.56) and (3.61),

$$
\begin{aligned}
\left(T_{n}^{(2)}\right)^{2} & \leq\left(\tau_{n} \sum_{k=1}^{n} \int_{\Omega} \frac{a\left(\widehat{u}^{k}\right)^{2}}{\varepsilon_{n}+a\left(\widehat{u}^{k-1}\right)} \mathrm{d} \mu\right)\left(\tau_{n} \sum_{k=1}^{n} \int_{\Omega} \frac{\left(a\left(\widehat{u}^{k}\right)-a\left(\widehat{u}^{k-1}\right)\right)^{2}}{\varepsilon_{n}+a\left(\widehat{u}^{k-1}\right)} \mathrm{d} \mu\right) \\
& \leq 2 \tau_{n} C_{1} C_{2} .
\end{aligned}
$$

We then follow the proof of (3.35) in Lemma 3.9, and we obtain the existence of an absolute constant $C_{0}$ such that

$$
\begin{aligned}
& \left\|\widehat{u}_{n}(t)-u(t)\right\|^{2} \\
& \leq C_{0}\left(C_{2} \tau_{n}+T\left\|f_{n}-f\right\|_{L^{2}(0, T ; H)}^{2}+\left\|\widehat{u}_{n}^{0}-u^{0}\right\|^{2}+\widehat{\mathcal{R}}_{u}\left(v_{n}\right)\right. \\
& \left.\quad+\varepsilon_{n} \mu(\Omega) T+\left(C_{2} \frac{\tau_{n}}{\varepsilon_{n}}\right)^{1 / 2}\left\|a\left(v_{n}\right)\right\|_{L^{2}\left(0, T ; L_{\mu}^{2}(\Omega)\right)}+\left(C_{1} C_{2} \tau_{n}\right)^{1 / 2}\right),
\end{aligned}
$$

for all $t \in[0, T]$, where $f_{n}(t)$ is defined by $(3.15)$ and $\widehat{\mathcal{R}}_{u}\left(v_{n}\right)$ is defined by

$$
\widehat{\mathcal{R}}_{u}\left(v_{n}\right)=\left(C_{2}^{1 / 2}+\|f\|_{L^{2}(0, T ; H)}\right)\left\|v_{n}-u\right\|_{L^{2}(0, T ; H)}+\int_{0}^{T}\left(\psi\left(v_{n}(t)\right)-\psi(u(t))\right)^{+} \mathrm{d} t .
$$

Let now $\eta>0$. As in the proof of Theorem 3.11, we can take $M \in \mathbb{N}^{\star}$ such that for some $t_{i} \in I_{i}=[(i-1) T / M, i T / M]$, one has (3.44) and (3.45). Then since $u\left(t_{i}\right) \in B$, one can use (3.52), and find $\varphi_{i} \in H_{1}$ such that

$$
\left\|\varphi_{i}-u\left(t_{i}\right)\right\|+\left(\psi\left(\varphi_{i}\right)-\psi\left(u\left(t_{i}\right)\right)\right)^{+} \leq \eta
$$


Define then

$$
\left.\left.\varphi(t)=\varphi_{i} \text { for all } t \in\right](i-1) T / M, i T / M\right], i=1, \ldots, M, \quad \varphi(0)=\varphi_{1} .
$$

Applying (3.53), one can find for all $i=1, \ldots, M$ some $v_{n}^{i} \in \widehat{H}_{n}$ such that

$$
\left\|v_{n}^{i}-\varphi_{i}\right\|+\left(\int a\left(v_{n}^{i}-\varphi_{i}\right)^{2} \mathrm{~d} \mu\right)^{1 / 2} \longrightarrow 0, \text { as } n \rightarrow \infty .
$$

This implies in particular that $\psi\left(v_{n}^{i}\right)-\psi\left(\varphi_{i}\right)=\int\left(a\left(v_{n}^{i}\right)-a\left(\varphi_{i}\right)\right) d \mu \rightarrow 0$. Define $v_{n}$ by

$$
\left.\left.v_{n}(t)=v_{n}^{i} \text { for all } t \in\right](i-1) T / M, i T / M\right], i=1, \ldots, M, \quad v_{n}(0)=v_{n}^{1} .
$$

Then

$$
\sup _{n} \sup _{t \in[0, T]} \int a\left(v_{n}(t)\right)^{2} \mathrm{~d} \mu<\infty .
$$

Therefore, passing to the limit in (3.62) as $n \rightarrow \infty$, we get

$$
\limsup _{n \rightarrow \infty} \sup _{t \in[0, T]}\left\|\widehat{u}_{n}(t)-u(t)\right\|^{2} \leq C_{0} \widehat{\mathcal{R}}_{u}(\varphi) \leq C \eta
$$

where $C$ depends only on $G,\|f\|_{L^{2}(0, T ; H)}, T$. This being true for all $\eta>0$, we conclude that

$$
\limsup _{n \rightarrow \infty} \sup _{t \in[0, T]}\left\|\widehat{u}_{n}(t)-u(t)\right\|^{2}=0
$$

showing the convergence of the scheme. The proof that $\psi\left(\widehat{u}_{n}\right)$ converges in $L^{1}(] 0, T[)$ to $\psi(u)$ uses a similar procedure, following the proof of (3.36) in Lemma 3.9.

Remark 3.15 Even if the implicit linearized algorithm (3.51) looks very simple in its implementation, it must be said that this simplicity hides indeed a very slow rate of convergence, obtained as $(\tau / \varepsilon)^{1 / 4}$ in a weak sense. This is much slower than the rate of Theorem 3.12 and Remark 3.13 (estimates (3.35), (3.36), (3.47), (3.48)). However, for solving (3.46), which is a nonlinear problem on $\widehat{u}_{\varepsilon}^{k}$, we have to use the iteration procedure (2.43) at each time step, which can be written

$$
\begin{aligned}
& \widehat{u}_{\varepsilon}^{k(j)} \in \widehat{H}, \\
& \left\langle\frac{\widehat{u}_{\varepsilon}^{k(j)}-\widehat{u}_{\varepsilon}^{k-1}}{\tau}, w\right\rangle+\int_{\Omega} \frac{A\left(\widehat{u}_{\varepsilon}^{k(j)}, w\right)(x)}{\varepsilon+a\left(\widehat{u}_{\varepsilon}^{k(j-1)}\right)(x)} \mathrm{d} \mu=\left\langle f^{k}, w\right\rangle, \forall w \in \widehat{H}, \forall j \in \mathbb{N}^{\star}, \\
& \widehat{u}_{\varepsilon}^{k(0)}=\widehat{u}_{\varepsilon}^{k-1},
\end{aligned}
$$

for $k=1, \ldots, n$. This can be extremely costly. In practice a strategy which is intermediate between the two algorithms is to perform only a few iterations in (3.63) (a single iteration corresponding to the regularized linearized implicit method (3.51)). Then the ratio accuracy versus cost is improved. 


\section{Numerical examples}

\subsection{Steady case}

We consider the framework of Section 2.2, and the total variation flow of example 2.11. We take $\Omega=] 0,1[$ and $\alpha=1$. We consider the function $f$ defined by

$$
f(x)=1000\left(x-\frac{1}{10}\right)\left(\frac{1}{2}-x\right)\left(x-\frac{2}{3}\right), \forall x \in[0,1] .
$$

Let us consider $0<x_{1}<x_{2}<x_{3}<x_{4}<1$ (see Figure 1) such that

$$
\begin{aligned}
& \int_{0}^{x_{1}}\left(f(s)-f\left(x_{1}\right)\right) \mathrm{d} s=1, \\
& f\left(x_{2}\right)=f\left(x_{3}\right), \\
& \int_{x_{2}}^{x_{3}}\left(f(s)-f\left(x_{2}\right)\right) \mathrm{d} s=0, \\
& \int_{x_{4}}^{1}\left(f(s)-f\left(x_{4}\right)\right) \mathrm{d} s=-1 .
\end{aligned}
$$

One can check that there exists a solution to these equations which is such that $x_{1} \simeq 0.0781928$, $x_{2} \simeq 0.1309924, x_{3} \simeq 0.7134521$ and $x_{4} \simeq 0.9501621$. We then define the function $u$ by

$$
\begin{aligned}
& u(x)=f\left(x_{1}\right), \forall x \in\left[0, x_{1}\right] \\
& u(x)=f(x), \forall x \in\left[x_{1}, x_{2}\right] \\
& u(x)=f\left(x_{2}\right)=f\left(x_{3}\right), \forall x \in\left[x_{2}, x_{3}\right] \\
& u(x)=f(x), \forall x \in\left[x_{3}, x_{4}\right] \\
& u(x)=f\left(x_{4}\right), \forall x \in\left[x_{4}, 1\right] .
\end{aligned}
$$

We can then prove, using Remark 2.4, that this function $u$ is the exact solution to (2.6) in this case. Indeed, on one hand we have

$$
\int_{0}^{1}\left(u(s)^{2}+\left|u^{\prime}(s)\right|\right) \mathrm{d} s=\int_{0}^{1} f(s) u(s) \mathrm{d} s,
$$

since $f$ is decreasing on $\left[x_{1}, x_{2}\right]$ and on $\left[x_{3}, x_{4}\right]$, which leads to

$$
\int_{0}^{1}\left|u^{\prime}(s)\right| \mathrm{d} s=f\left(x_{1}\right)-f\left(x_{4}\right),
$$

and since

$$
\begin{aligned}
& \int_{0}^{1}\left(f(s) u(s)-u(s)^{2}\right) \mathrm{d} s \\
= & \int_{0}^{x_{1}}\left(f(s)-f\left(x_{1}\right)\right) f\left(x_{1}\right) \mathrm{d} s+\int_{x_{2}}^{x_{3}}\left(f(s)-f\left(x_{2}\right)\right) f\left(x_{2}\right) \mathrm{d} s \\
& +\int_{x_{4}}^{1}\left(f(s)-f\left(x_{4}\right)\right) f\left(x_{4}\right) \mathrm{d} s \\
= & f\left(x_{1}\right)-f\left(x_{4}\right) .
\end{aligned}
$$

Defining $\mu(x)=\int_{0}^{x}(f(s)-u(s)) \mathrm{d} s$, we get that

$$
\begin{aligned}
& \mu(0)=\mu(1)=0, \\
& |\mu(x)| \leq 1, \forall x \in[0,1],
\end{aligned}
$$


which leads to

$$
\forall v \in H^{1}(] 0,1[), \int_{0}^{1}\left|v^{\prime}(s)\right| \mathrm{d} s \geq-\int_{0}^{1} \mu(s) v^{\prime}(s) \mathrm{d} s=\int_{0}^{1}(f(s)-u(s)) v(s) \mathrm{d} s .
$$

Gathering the two above equations, we get that $u \in H^{1}(] 0,1[)$ is such that

$$
\begin{aligned}
\forall v \in H^{1}(] 0,1[), & \int_{0}^{1}\left(u(s)(v(s)-u(s))+\left|v^{\prime}(s)\right|-\left|u^{\prime}(s)\right|\right) \mathrm{d} s \\
& \geq \int_{0}^{1} f(s)(v(s)-u(s)) \mathrm{d} s,
\end{aligned}
$$

which implies (2.6).

Let us now check that the numerical method considered in Lemmas 2.13 and 2.14 well approximates the function $u$.

We consider that $\widehat{H}$ is spanned by the $P^{1}$ finite element basis, using a constant space step $h=1 / 2000$. We take $\varepsilon=10^{-6}$ in (2.43), and $k=10000$ iterations of the algorithm (2.43), that provide $\left\|u^{(k)}-u^{(k-1)}\right\|_{\infty} /(\max (u)-\min (u)) \simeq 0.00002$. We get that $\left\|u^{(k)}-u\right\|_{\infty} /(\max (u)-$ $\min (u)) \simeq 0.007$, which is acceptable in this case. This is confirmed by Figure 1, where the three functions, $u, u^{(k)}$ and $f$ are drawn.

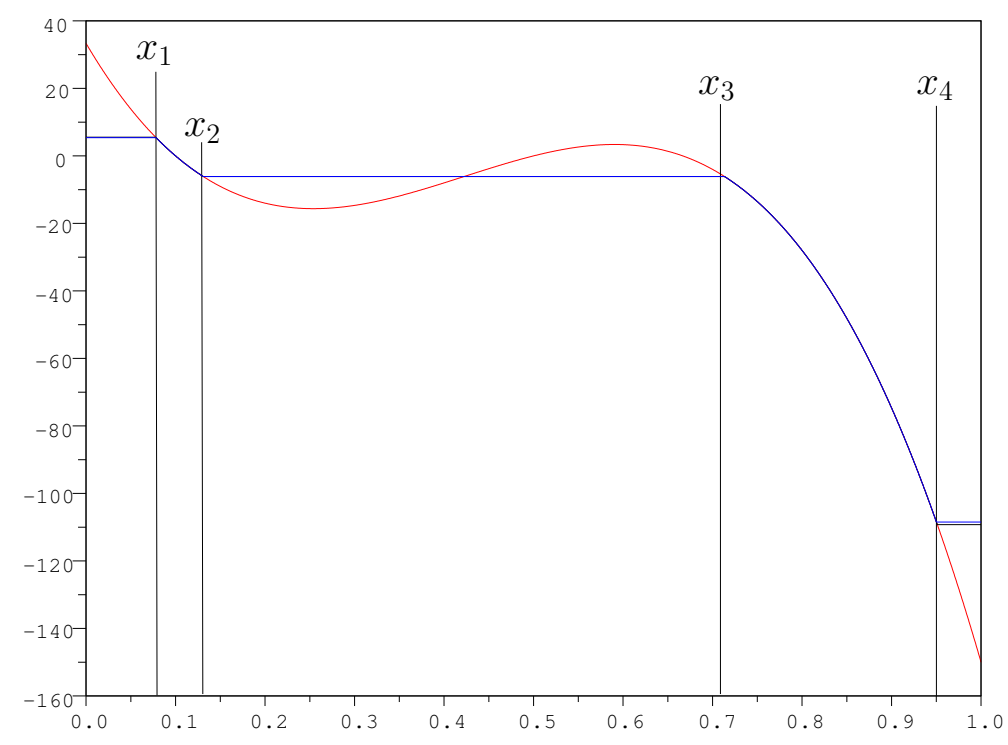

Figure 1: Approximate solution (blue), exact solution (black) and right-hand side (red).

\subsection{Transient case}

We again consider the total variation flow of example 2.11 with $\Omega=] 0,1[$. We consider the framework of subsection 3.4 , we let $f=0$ and

$$
u^{0}(x)=1000\left(x-\frac{1}{10}\right)\left(\frac{1}{2}-x\right)\left(x-\frac{2}{3}\right), \forall x \in[0,1] .
$$


We consider the following data in the scheme (3.51): $\widehat{H}$ is the $P^{1}$ finite element approximation with constant space step equal to $h=1 / 200, \tau=0.001, \varepsilon=0.01$. We show in Figure 2 the approximate solution at different times. It is then possible to check that Figure 2 provides an

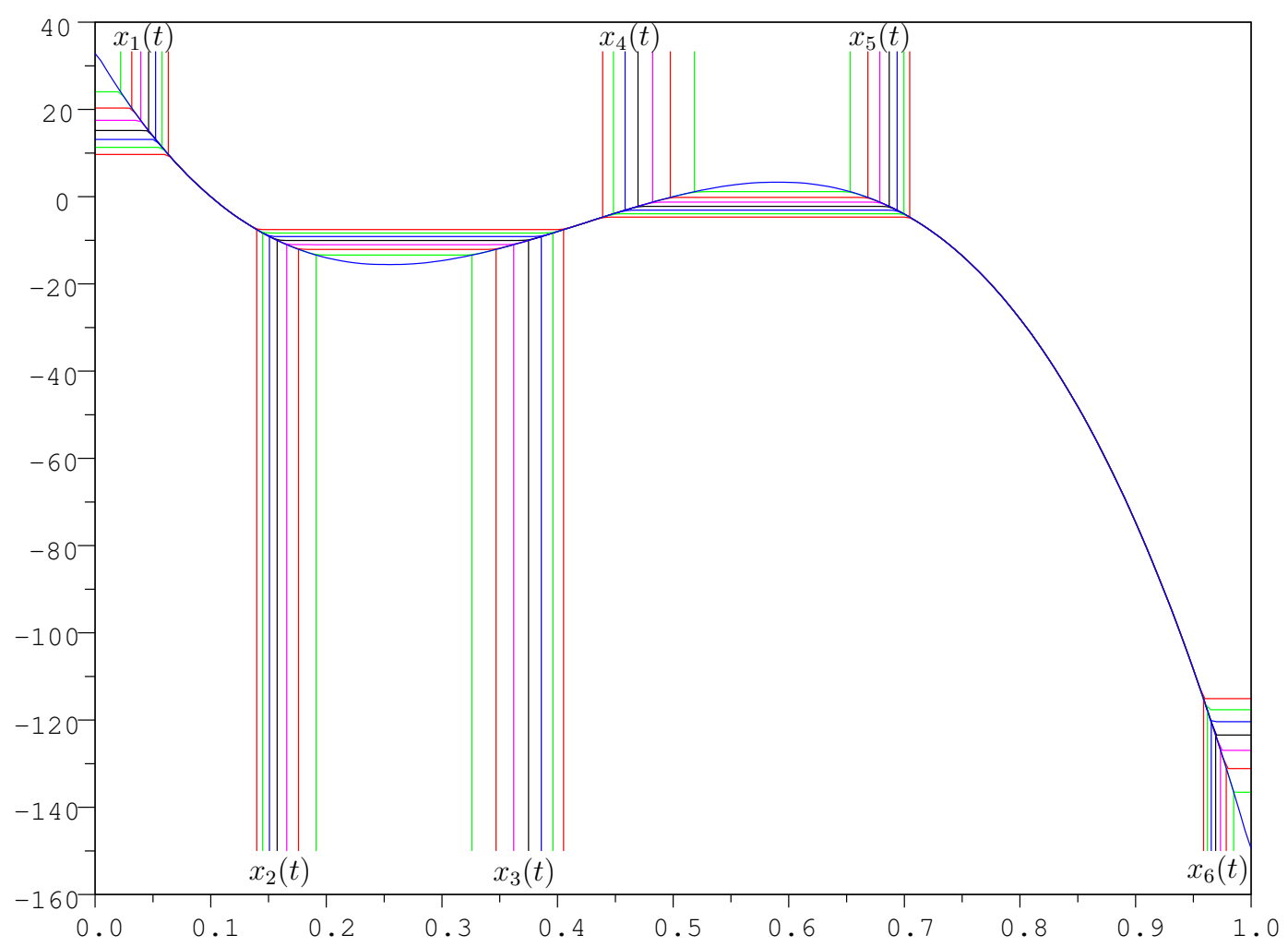

Figure 2: Initial solution (blue) and approximate solutions at times 0.1 (green), 0.2 (red), 0.3 (magenta), 0.4 (black), 0.5 (blue), 0.6 (green), 0.7 (red). The vertical lines are approximate values at the same times of $x_{i}(t), i=1, \ldots, 6$.

accurate approximation of the analytical solution of the problem, at least for small times. Let us give the ordinary differential equations leading to the definition of this analytical solution. We denote by $y_{1}=(76-\sqrt{916}) / 180 \simeq 0.2540806$ and $y_{2}=(76+\sqrt{916}) / 180 \simeq 0.5903638$ the two roots of the equation $\nabla u^{0}(x)=0$. The analytical solution is given for $t$ sufficiently small by $0<x_{1}(t)<x_{2}(t)<x_{3}(t)<x_{4}(t)<x_{5}(t)<x_{6}(t)<1$ (see Figure 2) such that, for all $t>0$ small enough such that $x_{3}(t)<x_{4}(t)$,

$$
\begin{gathered}
x_{1}(0)=0 \text { and } \partial_{t}\left(u^{0}\left(x_{1}(t)\right)\right)=-1 / x_{1}(t), \\
x_{2}(0)=x_{3}(0)=y_{1}, u^{0}\left(x_{2}(t)\right)=u^{0}\left(x_{3}(t)\right), \\
\partial_{t}\left(u^{0}\left(x_{3}(t)\right)\right)=2 /\left(x_{3}(t)-x_{2}(t)\right), \\
x_{4}(0)=x_{5}(0)=y_{2}, u^{0}\left(x_{4}(t)\right)=u^{0}\left(x_{5}(t)\right), \\
\partial_{t}\left(u^{0}\left(x_{5}(t)\right)\right)=-2 /\left(x_{5}(t)-x_{4}(t)\right), \\
x_{6}(0)=1 \text { and } \partial_{t}\left(u^{0}\left(x_{6}(t)\right)\right)=1 /\left(1-x_{6}(t)\right) .
\end{gathered}
$$


Note that the above system is not well posed at $t=0$, since $\left(u^{0}\right)^{\prime}\left(y_{1}\right)=\left(u^{0}\right)^{\prime}\left(y_{2}\right)=0$. We then define the function $u(t, x)$ by

$$
\begin{array}{llrl}
u(t, x) & =u^{0}\left(x_{1}(t)\right), & & \forall x \in\left[0, x_{1}(t)\right], \\
u(t, x) & =u^{0}(x), & & \forall x \in\left[x_{1}(t), x_{2}(t)\right], \\
u(t, x) & =u^{0}\left(x_{2}(t)\right)=u^{0}\left(x_{3}(t)\right), & & \forall x \in\left[x_{2}(t), x_{3}(t)\right], \\
u(t, x) & =u^{0}(x), & & \forall x \in\left[x_{3}(t), x_{4}(t)\right], \\
u(t, x) & =u^{0}\left(x_{4}(t)\right)=u^{0}\left(x_{5}(t)\right), & & \forall x \in\left[x_{4}(t), x_{5}(t)\right], \\
u(t, x) & =u^{0}(x), & & \forall x \in\left[x_{5}(t), x_{6}(t)\right], \\
u(t, x) & =u^{0}\left(x_{6}(t)\right), & & \forall x \in\left[x_{6}(t), 1\right] .
\end{array}
$$

The function $u$ verifies, for $t$ small enough such that $x_{3}(t)<x_{4}(t)$,

$$
\begin{aligned}
& \int_{0}^{1}\left(\partial_{t} u(t, x) u(t, x)+|\nabla u(t, x)|\right) \mathrm{d} x \\
= & -u^{0}\left(x_{1}(t)\right) \frac{x_{1}(t)}{x_{1}(t)}+u^{0}\left(x_{1}(t)\right)-u^{0}\left(x_{2}(t)\right)+2 u^{0}\left(x_{2}(t)\right) \frac{x_{3}(t)-x_{2}(t)}{x_{3}(t)-x_{2}(t)} \\
& +u^{0}\left(x_{4}(t)\right)-u^{0}\left(x_{3}(t)\right)-2 u^{0}\left(x_{4}(t)\right) \frac{x_{5}(t)-x_{4}(t)}{x_{5}(t)-x_{4}(t)} \\
& +u^{0}\left(x_{5}(t)\right)-u^{0}\left(x_{6}(t)\right)+u^{0}\left(x_{6}(t)\right) \frac{1-x_{6}(t)}{1-x_{6}(t)} \\
= & 0
\end{aligned}
$$

We then denote

$$
\mu(t, x)=\int_{0}^{x} \partial_{t} u(t, s) \mathrm{d} s .
$$

We remark that $\mu(t, 1)=1-2+2-1=0$ and it is easy to check that $-1 \leq \mu(t, x) \leq 1$ for all $x \in[0,1]$. Therefore we have

$$
\forall v \in H^{1}(] 0,1[), \int_{0}^{1}\left|v^{\prime}(x)\right| \mathrm{d} x \geq \int_{0}^{1} \mu(t, x) v^{\prime}(x) \mathrm{d} x=-\int_{0}^{1} \partial_{t} u(t, x) v(x) \mathrm{d} x .
$$

Gathering the above equations, we get that $u$ is such that

$$
\forall v \in H^{1}(] 0,1[), \int_{0}^{1}\left(\partial_{t} u(t, x)(v(x)-u(t, x))+|\nabla v(x)|-|\nabla u(t, x)|\right) \mathrm{d} x \geq 0,
$$

which proves that $u$ is the analytical solution of the problem. Therefore, in order to assess the accuracy of the numerical scheme, we have plotted on Figure 2 vertical lines at approximations of abscissae $x_{i}(t), i=1, \ldots, 6, t=0.1,0.2,0.3,0.4,0.5,0.6,0.7$, obtained using the following algorithms:

$$
x_{1} \rightarrow 0, \quad t_{1} \rightarrow 0
$$

while $t_{1}<T$

$$
\begin{aligned}
& x_{1} \rightarrow x_{1}+\delta x \\
& t_{1} \rightarrow t_{1}-\delta x\left(u^{0}\right)^{\prime}\left(x_{1}\right) x_{1}
\end{aligned}
$$$$
\text { endwhile }
$$

$x_{2} \rightarrow y_{1}, \quad x_{3} \rightarrow y_{1}, \quad t_{2} \rightarrow 0, \quad t_{3} \rightarrow 0$

while $t_{2}<T$ and $t_{3}<T$

if $t_{2}<t_{3}$ then

$$
\begin{aligned}
& x_{2} \rightarrow x_{2}-\delta x \\
& t_{2} \rightarrow t_{2}-\delta x\left(u^{0}\right)^{\prime}\left(x_{2}\right)\left(x_{3}-x_{2}\right) / 2
\end{aligned}
$$

else

$$
\begin{aligned}
& x_{3} \rightarrow x_{3}+\delta x \\
& t_{3} \rightarrow t_{3}+\delta x\left(u^{0}\right)^{\prime}\left(x_{3}\right)\left(x_{3}-x_{2}\right) / 2
\end{aligned}
$$$$
\text { endif }
$$

endwhile 
The approximation of $x_{6}$ is similar to that of $x_{1}$, and that of $x_{4}$ and $x_{5}$ is similar to that of $x_{2}$ and $x_{3}$. We then numerically check that these algorithms provide accurate approximations of (4.1) (in particular, $u^{0}\left(x_{2}(t)\right)$ remains close to $u^{0}\left(x_{3}(t)\right)$, and $u^{0}\left(x_{4}(t)\right)$ remains close to $u^{0}\left(x_{5}(t)\right)$, setting $\left.\delta x=10^{-4}\right)$.

\section{Conclusion}

We have introduced numerical approximations by conforming methods and regularization, for a general class of steady or time-dependent variational problems that include the total variation flow and the inviscid Bingham flow. We have proved their convergence, generalizing the results of $[13,14]$, and shown the accuracy of the approximation for the total variation flow problem on a one-dimensional analytic solution.

In general, the lack of viscosity generates a big loss of accuracy in the regions where the multivalued aspect of these systems takes effect. This point appears in our error estimates in Lemma 2.15 , where the number of iterations may be very large if $\varepsilon$ is small, and in Theorem 3.14, where the rate of convergence is very weak, in $(\tau / \varepsilon)^{1 / 4}$. The practical aspects of the method for inviscid incompressible Bingham fluid flows are evaluated in [21, 19]. A related work is [5], using the augmented Lagrangian method.

\section{Appendix}

Lemma 5.1 Let $\varepsilon>0$ be given and let $F_{\varepsilon}: \mathbb{R}^{+} \rightarrow \mathbb{R}^{+}$be defined by

$$
\forall z \in \mathbb{R}^{+}, F_{\varepsilon}(z)=\int_{0}^{z} \frac{s}{\varepsilon+s} \mathrm{~d} s=z-\varepsilon \log \frac{\varepsilon+z}{\varepsilon} .
$$

Then the following properties hold:

$$
\begin{gathered}
\forall z \in \mathbb{R}^{+}, \quad 0 \leq F_{\varepsilon}(z) \leq z, \\
\forall z \in \mathbb{R}^{+}, \quad F_{\varepsilon}(z) \geq(z-\varepsilon)(1-\log 2), \\
\forall c, d \in \mathbb{R}^{+}, F_{\varepsilon}(d)-F_{\varepsilon}(c)+\frac{(d-c)^{2}}{2(\varepsilon+c)} \leq \frac{d}{\varepsilon+c}(d-c),
\end{gathered}
$$

and

$$
\forall c, d \in \mathbb{R}^{+}, F_{\varepsilon}(d)-F_{\varepsilon}(c) \leq \frac{d}{\varepsilon+d}(d-c)
$$

Finally, one has

$$
\forall c, d \in \mathbb{R}^{+}, d-c-\varepsilon \leq \frac{d}{\varepsilon+d}(d-c)
$$

and

$$
\forall c, d, e \in \mathbb{R}^{+}, \frac{d}{\varepsilon+c}(e-d) \leq e-d+\varepsilon+\frac{|d-c|}{\varepsilon+c}(e+d) .
$$

Proof. The first property (5.2) is obvious from the integral definition of $F_{\varepsilon}$. We then observe that

$$
z \log 2-\varepsilon \log \frac{\varepsilon+z}{\varepsilon} \geq 0, \forall z \in[\varepsilon, \infty)
$$


which implies (5.3). We then set, for $c, d \in \mathbb{R}_{+}, \Phi_{c}(d)=\frac{d}{\varepsilon+c}(d-c)-\frac{(d-c)^{2}}{2(\varepsilon+c)}-\int_{c}^{d} \frac{z \mathrm{~d} z}{\varepsilon+z}$. We have $\Phi_{c}(c)=0$, and $\Phi_{c}^{\prime}(d)=\frac{d}{\varepsilon+c}-\frac{d}{\varepsilon+d}$, whose sign is that of $d-c$. Hence $\Phi_{c}(d) \geq 0$, which proves (5.4). We then set, for $c, d \in \mathbb{R}_{+}, \widehat{\Phi}_{c}(d)=\frac{d}{\varepsilon+d}(d-c)-\int_{c}^{d} \frac{z \mathrm{~d} z}{\varepsilon+z}$. We have $\widehat{\Phi}_{c}(c)=0$, and $\widehat{\Phi}_{c}^{\prime}(d)=\frac{\varepsilon}{(\varepsilon+d)^{2}}(d-c)$, whose sign is that of $d-c$. Hence $\widehat{\Phi}_{c}(d) \geq 0$, which proves (5.5).

Finally, the proof of (5.6) is obtained by developing the expressions, as well as that of (5.7), which results from (5.6) and from

$$
\frac{d}{\varepsilon+c}(e-d)-\frac{d}{\varepsilon+d}(e-d)=\frac{d}{\varepsilon+d} \frac{d-c}{\varepsilon+c}(e-d) \leq \frac{|d-c|}{\varepsilon+c}(e+d) .
$$

Remark 5.2 One can perform the same analysis if $\varepsilon+a(u)$ is replaced by $\sqrt{\varepsilon^{2}+a(u)^{2}}$ in (2.31). Then $F_{\varepsilon}(z)=\sqrt{\varepsilon^{2}+z^{2}}-\varepsilon$.

We now state and prove the following compactness result for the sake of completeness.

Lemma 5.3 (Weak version of Ascoli's theorem) Let $T>0$ be given and let $H$ be a Hilbert space. Let $\left(u_{n}\right)_{n \in \mathbb{N}}$ be a sequence of functions from $[0, T]$ to $H$, such that there exists $C_{\infty} \geq 0$ with

$$
\left\|u_{n}(t)\right\| \leq C_{\infty}, \forall n \in \mathbb{N}, \forall t \in[0, T] .
$$

We also assume that there exists a sequence $\left(\tau_{n}\right)_{n \in \mathbb{N}}$ with $\tau_{n} \geq 0$ and $\tau_{n} \rightarrow 0$ as $n \rightarrow \infty$, and a constant $C_{2} \geq 0$ such that

$$
\left\|u_{n}\left(t_{2}\right)-u_{n}\left(t_{1}\right)\right\| \leq C_{2}^{1 / 2}\left(\left|t_{2}-t_{1}\right|+\tau_{n}\right)^{1 / 2}, \forall n \in \mathbb{N}, \forall t_{1}, t_{2} \in[0, T] .
$$

Then there exists $u \in C^{0}([0, T] ; H)$ and a subsequence of $\left(u_{n}\right)_{n \in \mathbb{N}}$, again denoted $\left(u_{n}\right)_{n \in \mathbb{N}}$, such that, for all $t \in[0, T], u_{n}(t)$ converges to $u(t)$ for the weak topology of $H$, and for all $v \in H$, $\left\langle u_{n}(t), v\right\rangle$ converges uniformly with respect to $t \in[0, T]$ to $\langle u(t), v\rangle$.

Proof. The proof follows that of Ascoli's theorem. Let $\left(t_{p}\right)_{p \in \mathbb{N}}$ be a dense sequence in $[0, T]$. In view of (5.8), for each $p \in \mathbb{N}$, we may extract from $\left(u_{n}\left(t_{p}\right)\right)_{n \in \mathbb{N}}$ a subsequence which is convergent to some element of $H$ for the weak topology of $H$. Using the diagonal method, we can find a strictly increasing function $\varphi: \mathbb{N} \rightarrow \mathbb{N}$, such that $\left(u_{\varphi(n)}\left(t_{p}\right)\right)_{n \in \mathbb{N}}$ is weakly convergent in $H$ for all $p \in \mathbb{N}$. For any $t \in[0, T]$ and $v \in H$, we then prove that the sequence $\left(\left\langle u_{\varphi(n)}(t), v\right\rangle\right)_{n \in \mathbb{N}}$ is a Cauchy sequence. Indeed, let $\varepsilon>0$ be given. We choose $p \in \mathbb{N}$ such that $\left|t-t_{p}\right| \leq \varepsilon^{2}$. Since $\left(\left\langle u_{\varphi(n)}\left(t_{p}\right), v\right\rangle\right)_{n \in \mathbb{N}}$ is a Cauchy sequence, we can find $n_{0} \in \mathbb{N}$ such that, for $k, l \geq n_{0}$,

$$
\left|\left\langle u_{\varphi(k)}\left(t_{p}\right)-u_{\varphi(l)}\left(t_{p}\right), v\right\rangle\right| \leq \varepsilon,
$$

and such that $\tau_{\varphi(k)}, \tau_{\varphi(l)} \leq \varepsilon^{2}$. We then get, using (5.9),

$$
\left|\left\langle u_{\varphi(k)}(t)-u_{\varphi(l)}(t), v\right\rangle\right| \leq C_{2}^{1 / 2}\|v\|\left(\left(\left|t-t_{p}\right|+\tau_{\varphi(k)}\right)^{1 / 2}+\left(\left|t-t_{p}\right|+\tau_{\varphi(l)}\right)^{1 / 2}\right)+\varepsilon,
$$

which gives

$$
\left|\left\langle u_{\varphi(k)}(t)-u_{\varphi(l)}(t), v\right\rangle\right| \leq\left(22^{1 / 2} C_{2}^{1 / 2}\|v\|+1\right) \varepsilon
$$


This proves that the sequence $\left(\left\langle u_{\varphi(n)}(t), v\right\rangle\right)_{n \in \mathbb{N}}$ converges. It is clear that its limit is a linear function of $v$. Since, from (5.8), we have

$$
\left|\left\langle u_{\varphi(n)}(t), v\right\rangle\right| \leq C_{\infty}\|v\|,
$$

we get by Riesz' theorem the existence of $u(t) \in H$ such that $\left(u_{\varphi(n)}(t)\right)_{n \in \mathbb{N}}$ converges to $u(t)$ for the weak topology of $H$. From (5.9), we have

$$
\left|\left\langle u_{n}\left(t_{2}\right)-u_{n}\left(t_{1}\right), v\right\rangle\right| \leq C_{2}^{1 / 2}\left(\left|t_{2}-t_{1}\right|+\tau_{n}\right)^{1 / 2}\|v\|, \forall n \in \mathbb{N}, \forall t_{1}, t_{2} \in[0, T], \forall v \in H .
$$

Passing to the limit $n \rightarrow \infty$ in the above equation, we get

$$
\left|\left\langle u\left(t_{2}\right)-u\left(t_{1}\right), v\right\rangle\right| \leq C_{2}^{1 / 2}\left|t_{2}-t_{1}\right|^{1 / 2}\|v\|, \forall t_{1}, t_{2} \in[0, T], \forall v \in H
$$

thus

$$
\left\|u\left(t_{2}\right)-u\left(t_{1}\right)\right\| \leq C_{2}^{1 / 2}\left|t_{2}-t_{1}\right|^{1 / 2}, \forall t_{1}, t_{2} \in[0, T],
$$

showing that $u \in C^{0}([0, T] ; H)$. Finally, fix $v \in H$, and let $\varepsilon>0$. Then there is a finite $P \in \mathbb{N}$ such that $\left.[0, T] \subset \cup_{p=1}^{P}\right] t_{p}-\varepsilon^{2}, t_{p}+\varepsilon^{2}\left[\right.$. Then there exists $n_{1}$ such that for all $n \geq n_{1}$ one has $\tau_{n} \leq \varepsilon^{2}$ and for all $p=1, \ldots, P$,

$$
\left|\left\langle u_{n}\left(t_{p}\right)-u\left(t_{p}\right), v\right\rangle\right| \leq \varepsilon .
$$

Then for a time $t \in[0, T]$, one can find $p \leq P$ such that $\left|t-t_{p}\right| \leq \epsilon^{2}$. It follows that

$$
\begin{aligned}
\left|\left\langle u_{n}(t)-u(t), v\right\rangle\right| & \leq\left|\left\langle u_{n}(t)-u_{n}\left(t_{p}\right), v\right\rangle\right|+\left|\left\langle u(t)-u\left(t_{p}\right), v\right\rangle\right|+\varepsilon \\
& \leq 2\left(C_{2}\right)^{1 / 2}\|v\|\left(\left|t-t_{p}\right|+\tau_{n}\right)^{1 / 2}+\varepsilon \\
& \leq\left(22^{1 / 2} C_{2}^{1 / 2}\|v\|+1\right) \varepsilon,
\end{aligned}
$$

which proves the uniform convergence with respect to $t$.

We have the following approximation properties, partly stated in [17] and [22] without full proof.

Lemma 5.4 Let $\Omega \subset \mathbb{R}^{N}$, with $N \geq 1$, be an open bounded set, such that there exists a point $O \in \Omega$ with $\Omega$ strictly star-shaped with respect to $O$. Then

$$
\forall u \in L^{2}(\Omega) \cap B V(\Omega), \inf _{v \in C^{\infty}(\bar{\Omega})}\left(\|v-u\|_{L^{2}(\Omega)}^{2}+\left.\left|\|\nabla v\|_{L^{1}(\Omega)}-\right| u\right|_{B V(\Omega)} \mid\right)=0,
$$

and

$$
\begin{aligned}
& \forall u \in L^{2}(\Omega)^{N} \text { with } D u \in \mathcal{M}(\Omega), \operatorname{div} u=0, \\
& \inf _{v \in C^{\infty}(\bar{\Omega})^{N}, \operatorname{div} v=0}\left(\|v-u\|_{L^{2}(\Omega)}^{2}+\left.\left|\|D v\|_{L^{1}(\Omega)}-\right| D u\right|_{\mathcal{M}(\Omega)} \mid\right)=0 .
\end{aligned}
$$

Proof. Here, $\mathcal{M}(\Omega)$ denotes the space of finite measures over $\Omega$, and $D u=\left(\nabla u+(\nabla u)^{t}\right) / 2$. We only prove (5.12), since (5.13) is obtained very similarly, replacing (1.20), (1.21) by (1.15), (1.16) with $\nu=0$. Let us assume, without restricting the generality, that the point $O$ is the origin of $\mathbb{R}^{N}$. Let $u \in L^{2}(\Omega) \cap B V(\Omega)$ be given, and let $n \in \mathbb{N}$ be given. We define $\Omega_{n}$ by

$$
\Omega_{n}=\left\{x \in \mathbb{R}^{N}, \frac{1}{1-\frac{1}{n+2}} x \in \Omega\right\} \subset \Omega .
$$


Since $\Omega$ is strictly star-shaped with respect to $O$, one has $\partial \Omega_{n} \cap \partial \Omega=\emptyset$. Since $\Omega$ is bounded, we have that $a_{n}=\frac{1}{4} \mathrm{~d}\left(\partial \Omega_{n}, \partial \Omega\right)>0$. Moreover, $a_{n} \rightarrow 0$ as $n \rightarrow \infty$. For a mollifier $\rho \in$ $C_{c}^{\infty}\left(B(0,1), \mathbb{R}^{+}\right)$with integral equal to 1 , we define the function $\rho_{n}$ by $\rho_{n}(x)=\frac{1}{a_{n}^{N}} \rho\left(\frac{x}{a_{n}}\right)$. We then consider the function $u_{n} \in C^{\infty}\left(\overline{\Omega_{n}}\right)$ defined by

$$
u_{n}(x)=\int_{\Omega} u(y) \rho_{n}(x-y) \mathrm{d} y, \forall x \in \Omega_{n} .
$$

Let $\varphi \in C_{c}^{1}\left(\Omega_{n}\right)^{N}$, with $|\varphi(x)| \leq 1$, for all $x \in \Omega_{n}$. We have

$$
\begin{aligned}
\int_{\Omega_{n}} u_{n}(x) \operatorname{div} \varphi(x) \mathrm{d} x & =\int_{\Omega_{n}} \int_{\Omega} u(y) \rho_{n}(x-y) \mathrm{d} y \operatorname{div} \varphi(x) \mathrm{d} x \\
& =\int_{\Omega} u(y) \int_{\Omega_{n}} \rho_{n}(x-y) \operatorname{div} \varphi(x) \mathrm{d} x \mathrm{~d} y \\
& =\int_{\Omega} u(y) \operatorname{div} \varphi_{n}(y) \mathrm{d} y,
\end{aligned}
$$

where $\varphi_{n}$ denotes the function defined by

$$
\varphi_{n}(y)=\int_{\Omega_{n}} \rho_{n}(x-y) \varphi(x) \mathrm{d} x, \forall y \in \Omega
$$

We have $\varphi_{n} \in C_{c}^{1}(\Omega)^{N}$, with $\left|\varphi_{n}(y)\right| \leq 1$ for all $y \in \Omega$. Therefore, because of the characterization (1.20), (1.21) of the $B V$ seminorm,

$$
\int_{\Omega} u(y) \operatorname{div} \varphi_{n}(y) \mathrm{d} y \leq|u|_{B V(\Omega)} .
$$

Since this holds for all $\varphi \in C_{c}^{1}\left(\Omega_{n}\right)^{N}$ with $|\varphi(x)| \leq 1$, we get

$$
\left|u_{n}\right|_{B V\left(\Omega_{n}\right)} \leq|u|_{B V(\Omega)}
$$

and therefore $\lim \sup _{n \rightarrow \infty}\left|u_{n}\right|_{B V\left(\Omega_{n}\right)} \leq|u|_{B V(\Omega)}$. Reciprocally, let $\varphi \in C_{c}^{1}(\Omega)^{N}$, with $|\varphi(x)| \leq 1$, for all $x \in \Omega$. Since, for all $n \in \mathbb{N}$, we have that $\mathrm{d}\left(\partial \Omega_{n}, \partial \Omega\right) \leq \frac{1}{n+2} \mathrm{~d}(O, \partial \Omega)$, there exists $n_{0} \in \mathbb{N}$ such that, for all $n \geq n_{0}, \varphi \in C_{c}^{1}\left(\Omega_{n}\right)^{N}$. For such an $n$, one has

$$
\int_{\Omega_{n}} u_{n}(x) \operatorname{div} \varphi(x) \mathrm{d} x \leq\left|u_{n}\right|_{B V\left(\Omega_{n}\right)} .
$$

Since this holds for all $n \geq n_{0}$ and since $u_{n}$ converges to $u$ in $L^{2}$, one gets

$$
\int_{\Omega} u(x) \operatorname{div} \varphi(x) \mathrm{d} x \leq \liminf _{n \rightarrow \infty}\left|u_{n}\right|_{B V\left(\Omega_{n}\right)} .
$$

Since this holds for all $\varphi \in C_{c}^{1}(\Omega)^{N}$, with $|\varphi(x)| \leq 1$, we may take the supremum in the above inequality, which yields

$$
|u|_{B V(\Omega)} \leq \liminf _{n \rightarrow \infty}\left|u_{n}\right|_{B V\left(\Omega_{n}\right)} .
$$

Hence we conclude, gathering these results, that

$$
\lim _{n \rightarrow \infty}\left|u_{n}\right|_{B V\left(\Omega_{n}\right)}=|u|_{B V(\Omega)} .
$$


Let us now define, for $n \in \mathbb{N}$,

$$
\widetilde{u}_{n}(x)=u_{n}\left(\left(1-\frac{1}{n+2}\right) x\right), \forall x \in \Omega .
$$

Then $\widetilde{u}_{n} \in C^{\infty}(\bar{\Omega})$. Let $\varphi \in C_{c}^{1}(\Omega)^{N}$, with $|\varphi(x)| \leq 1$, for all $x \in \Omega$. We have

$$
\begin{aligned}
\int_{\Omega} \widetilde{u}_{n}(x) \operatorname{div} \varphi(x) \mathrm{d} x & =\int_{\Omega} u_{n}\left(\left(1-\frac{1}{n+2}\right) x\right) \operatorname{div} \varphi(x) \mathrm{d} x \\
& =\frac{1}{\left(1-\frac{1}{n+2}\right)^{N}} \int_{\Omega_{n}} u_{n}(y) \operatorname{div} \varphi\left(\frac{y}{1-\frac{1}{n+2}}\right) \mathrm{d} y .
\end{aligned}
$$

Denoting $\varphi_{n}$ the function defined by

$$
\varphi_{n}(y)=\varphi\left(\frac{y}{1-\frac{1}{n+2}}\right), \forall y \in \Omega_{n},
$$

we have $\varphi_{n} \in C_{c}^{1}\left(\Omega_{n}\right)^{N}$ with $\left|\varphi_{n}(y)\right| \leq 1$ for all $y \in \Omega_{n}$, and $\operatorname{div} \varphi_{n}(y)=\frac{1}{1-\frac{1}{n+2}} \operatorname{div} \varphi\left(\frac{y}{1-\frac{1}{n+2}}\right)$. Hence

$$
\begin{aligned}
\int_{\Omega} \widetilde{u}_{n}(x) \operatorname{div} \varphi(x) \mathrm{d} x & =\frac{1}{\left(1-\frac{1}{n+2}\right)^{N-1}} \int_{\Omega_{n}} u_{n}(y) \operatorname{div} \varphi_{n}(y) \mathrm{d} y \\
& \leq \frac{1}{\left(1-\frac{1}{n+2}\right)^{N-1}}\left|u_{n}\right|_{B V\left(\Omega_{n}\right)} .
\end{aligned}
$$

We then get that

$$
\left|\widetilde{u}_{n}\right|_{B V(\Omega)} \leq \frac{1}{\left(1-\frac{1}{n+2}\right)^{N-1}}\left|u_{n}\right|_{B V\left(\Omega_{n}\right)} .
$$

We show in a similar way the converse inequality, thus

$$
\left|\widetilde{u}_{n}\right|_{B V(\Omega)}=\frac{1}{\left(1-\frac{1}{n+2}\right)^{N-1}}\left|u_{n}\right|_{B V\left(\Omega_{n}\right)} .
$$

With (5.14) we conclude that

$$
\lim _{n \rightarrow \infty}\left|\widetilde{u}_{n}\right|_{B V(\Omega)}=|u|_{B V(\Omega)} .
$$

Since $\widetilde{u}_{n}$ converges as well to $u$ in $L^{2}$, this concludes the proof of (5.12).

Lemma 5.5 Let $H$ be a Hilbert space, with norm $\|\cdot\|$, and let $\psi$ satisfy Hypothesis 2.6. Let $T>0$ be given, and let $B_{T}$ be defined as $B_{T}=\left\{v \in L^{2}(0, T ; H) ; \int_{0}^{T} \psi(v(t)) \mathrm{d} t<\infty\right\}$. Let $v \in B_{T}$, and let, for all $n \in \mathbb{N}, v_{n} \in B_{T}$ such that

$$
\lim _{n \rightarrow \infty}\left\|v_{n}-v\right\|_{L^{2}(0, T ; H)}=0, \quad \lim _{n \rightarrow \infty} \int_{0}^{T}\left(\psi\left(v_{n}(t)\right)-\psi(v(t))\right)^{+} \mathrm{d} t=0 .
$$

Then $\psi\left(v_{n}\right)-\psi(v) \rightarrow 0$ in $L^{1}(] 0, T[)$.

Proof. We first extract a subsequence of $\left(v_{n}\right)$ such that, for a.e. $\left.t \in\right] 0, T\left[, v_{n}(t) \rightarrow v(t)\right.$ in $H$. Using the lower semi-continuity of $\psi$ and Fatou's lemma,

$$
\int_{0}^{T} \psi(v) \leq \int_{0}^{T} \liminf \psi\left(v_{n}\right) \leq \liminf \int_{0}^{T} \psi\left(v_{n}\right) \leq \limsup \int_{0}^{T} \psi\left(v_{n}\right) .
$$


But since

$$
\int_{0}^{T} \psi\left(v_{n}\right)-\int_{0}^{T} \psi(v) \leq \int_{0}^{T}\left(\psi\left(v_{n}\right)-\psi(v)\right)^{+} \rightarrow 0,
$$

we deduce that $\lim \sup \int_{0}^{T} \psi\left(v_{n}\right) \leq \int_{0}^{T} \psi(v)$, and $\int_{0}^{T} \psi\left(v_{n}\right) \rightarrow \int_{0}^{T} \psi(v)$. Then

$$
\int_{0}^{T}\left|\psi\left(v_{n}\right)-\psi(v)\right|=2 \int_{0}^{T}\left(\psi\left(v_{n}\right)-\psi(v)\right)^{+}-\int_{0}^{T}\left(\psi\left(v_{n}\right)-\psi(v)\right) \rightarrow 0 .
$$

We can argue in this way for all subsequences of $\left(v_{n}\right)$, thus we conclude that the whole sequence converges.

\section{References}

[1] F. Andreu, C. Ballester, V. Caselles, and J. M. Mazón. Minimizing total variation flow. Differential Integral Equations, 14(3):321-360, 2001.

[2] V. Barbu. Nonlinear semigroups and differential equations in Banach spaces. Editura Academiei Republicii Socialiste România, Bucharest, 1976. Translated from the Romanian.

[3] V. Barbu. Nonlinear differential equations of monotone types in Banach spaces. Springer Monographs in Mathematics. Springer, New York, 2010.

[4] F. Bouchut, D. Doyen, and R. Eymard. Convection and total variation flow. IMA J. Num. Anal., 2013.

[5] D. Bresch, E. D. Fernández-Nieto, I. R. Ionescu, and P. Vigneaux. Augmented Lagrangian method and compressible visco-plastic flows: applications to shallow dense avalanches. In New directions in mathematical fluid mechanics, Adv. Math. Fluid Mech., pages 57-89. Birkhäuser Verlag, Basel, 2010.

[6] H. Brézis. Opérateurs maximaux monotones et semi-groupes de contractions dans les espaces de Hilbert. North-Holland Publishing Co., Amsterdam, 1973. North-Holland Mathematics Studies, No. 5. Notas de Matemática (50).

[7] H. Brézis. Functional analysis, Sobolev spaces and partial differential equations. Springer, New-York, Dordrecht, Heidelberg, London, 2010.

[8] G. Cohen. Convexité et optimisation. Polycopié Ecole Nationale des Ponts et Chaussées, Champs-sur-Marne, 2000.

[9] M. G. Crandall and T. M. Liggett. Generation of semi-groups of nonlinear transformations on general Banach spaces. Amer. J. Math., 93:265-298, 1971.

[10] M. G. Crandall and L. Tartar. Some relations between nonexpansive and order preserving mappings. Proc. Amer. Math. Soc., 78(3):385-390, 1980.

[11] J. Droniou. Intégration et Espaces de Sobolev à Valeurs Vectorielles. http://www-gm3.univmrs.fr/polys/gm3-02/index.html. Polycopié gm3-02 de l'Ecole Doctorale de Maths-Info, Marseille, 2001. 
[12] G. Duvaut and J.-L. Lions. Inequalities in mechanics and physics. Springer-Verlag, Berlin, 1976. Translated from the French by C. W. John, Grundlehren der Mathematischen Wissenschaften, 219.

[13] X. Feng and A. Prohl. Analysis of total variation flow and its finite element approximations. M2AN Math. Model. Numer. Anal., 37(3):533-556, 2003.

[14] X. Feng, M. von Oehsen, and A. Prohl. Rate of convergence of regularization procedures and finite element approximations for the total variation flow. Numer. Math., 100(3):441-456, 2005 .

[15] R. Glowinski, J.-L. Lions, and R. Trémolières. Numerical analysis of variational inequalities, volume 8 of Studies in Mathematics and its Applications. North-Holland Publishing Co., Amsterdam, 1981. Translated from the French.

[16] R. Glowinski, J. Xu, and P. G. Ciarlet, editors. Handbook of numerical analysis. Vol. XVI. Special volume: Numerical Methods for Non-Newtonian Fluids, volume 16 of Handbook of Numerical Analysis. Elsevier/North-Holland, Amsterdam, 2011.

[17] E. Godlewski and P.-A. Raviart. Hyperbolic systems of conservation laws, volume 3/4 of Mathématiques \& Applications (Paris) [Mathematics and Applications]. Ellipses, Paris, 1991.

[18] J.-B. Hiriart-Urruty and C. Lemaréchal. Convex analysis and minimization algorithms. I, volume 305 of Grundlehren der Mathematischen Wissenschaften [Fundamental Principles of Mathematical Sciences]. Springer-Verlag, Berlin, 1993. Fundamentals.

[19] P.-Y. Lagrée, L. Staron, and S. Popinet. The granular column collapse as a continuum: validity of a two-dimensional Navier-Stokes model with a $\mu(I)$-rheology. J. Fluid Mech., 686:378-408, 2011.

[20] J.-L. Lions. Remarks on some nonlinear evolution problems arising in Bingham flows. In Proceedings of the International Symposium on Partial Differential Equations and the Geometry of Normed Linear Spaces (Jerusalem, 1972), volume 13, pages 155-172 (1973), 1972 .

[21] C. Lusso. Modelling and simulation of granular flows with fluid/solid transition with the Drucker-Prager model. PhD thesis. Université Paris-Est, Champs-sur-Marne, 2013.

[22] J. M. Mazón. The total variation flow. In Proceedings of minisemester on evolution of interfaces, Technical Report 145, pages 163-210, 2010.

[23] C. Nouar and A. Bottaro. Stability of the flow of a Bingham fluid in a channel: eigenvalue sensitivity, minimal defects and scaling laws of transition. J. Fluid Mech., 642:349-372, 2010 . 